\title{
Transposition
}

Musique et Sciences Sociales

$7 \mid 2018$

Le prix de la musique

\section{Les premières éditions de pièces de clavecin en France, œuvres musicales et économie}

\section{Chloé Dos Reis}

\section{(2) OpenEdition}

1 Journals

\section{Édition électronique}

URL : http://journals.openedition.org/transposition/1810

DOI : 10.4000/transposition. 1810

ISSN : 2110-6134

Éditeur

CRAL - Centre de recherche sur les arts et le langage

\section{Référence électronique}

Chloé Dos Reis, «Les premières éditions de pièces de clavecin en France, œuvres musicales et économie », Transposition [En ligne], 7 | 2018, mis en ligne le 15 septembre 2018, consulté le 10 décembre 2020. URL : http://journals.openedition.org/transposition/1810 ; DOI : https://doi.org/ 10.4000/transposition.1810

Ce document a été généré automatiquement le 10 décembre 2020.

La revue Transposition est mise à disposition selon les termes de la Licence Creative Commons Attribution - Partage dans les Mêmes Conditions 4.0 International. 


\title{
Les premières éditions de pièces de clavecin en France, œuvres musicales et économie
}

\author{
Chloé Dos Reis
}

\section{Introduction}

1 De 1670 à 1707, neuf compositeurs ont publié des pièces pour clavecin à Paris à compte d'auteur : Chambonnières (1670), Lebègue (1677 et 1687), Jacquet de la Guerre (1687 et 1707), d'Anglebert (1689), Marchand (1699 et 1702), Clérambault (1704), Dandrieu (1704), Le Roux (1705) et Rameau (1706). L'édition d'œuvres pour clavecin n'avait jamais été mise en place jusqu'alors. L'imprimeur Ballard, bénéficiant du privilège royal, ne vit pas l'intérêt de telles publications avant 1707. Comment expliquer l'apparition de cette nouvelle source musicale et son tel développement au début du XVIII ${ }^{\mathrm{e}}$ siècle ?

2 Le premier jalon représentatif de l'école française naissante est livré dans le manuscrit anversois de John Bull dans les années $1620^{1}$. La première édition pour clavecin de 1670 est donc postérieure de près de cinquante ans aux premiers manuscrits. Les caprices des artistes tels qu'on peut les lire dans les écrits des musicologues du début $d u x^{e}$ siècle ne sont pas l'unique raison de ces tardives publications :

Les virtuoses se souciaient peu d'éditer leur musique parce qu'elle représentait pour eux une propriété exclusive, dont seuls ils tiraient parti. [...] La composition et l'exécution se confondent encore. Les artistes gardent donc jalousement leur musique pour eux'.

L'histoire de l'imprimerie musicale en France et de ses contraintes imposées permet d'expliquer différemment ce décalage entre la pratique et l'édition. La famille Ballard, composée d'imprimeurs, est une véritable dynastie, allant de 1552 à la fin de l'Ancien Régime $^{3}$, gardant jalousement par privilège un monopole voué au luth ou au chant. Or, cette famille néglige aussi bien l'orgue, l'épinette et le clavicorde que la viole et la musique d'ensemble avec basse continue. Il semble pourtant que dans les années 1550, les 
associés Adrien Le Roy et Robert Ballard aient acheté des caractères pour imprimer une "petite tablature d'épinette » et une " grosse tablature d'épinette », mais ce matériel n'a jamais été utilisét ${ }^{4}$. Les seules musiques imprimées destinées au clavier sorties des presses françaises avant le premier quart du XVII ${ }^{\mathrm{e}}$ siècle furent les Hymnes de l'Église pour toucher sur l'orgue et le Magnificat de Jehan Titelouze, chez Ballard en 1623 et 1625. Du vivant de Louis XIV, trois membres de la famille Ballard ont exercé la typographie musicale: Robert III de 1639 à 1673, son fils Christophe de 1673 à 1715 et son frère Pierre III de 1695 à 1697. De 1640 à 1675 environ, Robert III Ballard occupe la presque totalité du marché de l'édition musicale. Les Ballard, et le roi, imposent leurs goûts à la France entière et ne consacrent rien au clavecin avant 1702. La technique utilisée est celle, assez rigide, de la typographie, dont le système fonctionnait par un procédé d'emboîtage de caractères mobiles constituant les pièces d'une espèce de puzzle. Cette technique rencontre vite des difficultés. Le facteur le plus important à avoir favorisé la perte de la forme losangée de la typographie tient à l'évolution de l'écriture musicale. Entre autres, l'adjonction massive d'ornements (surtout sous forme de signes, c'est-à-dire d'agréments) et le développement croissant de la virtuosité mirent en évidence les limites de cette technique. Or, la gravure, exercée d'abord par des artisans originaires du Nord de l'Europe ${ }^{5}$, permettait de dessiner la musique et de lui apporter les lignes et les courbes dont elle avait besoin pour s'exprimer par la notation. Le graveur pensait sa page de musique comme une entité graphique, puis, à l'aide d'outils (burin, poinçon, traceur de portée) composait, sur plaque de cuivre et surtout d'étain, qui est un matériel relativement tendre, la page de la partition ou de la partie séparée. La gravure était effectuée à l'envers ou en miroir afin que les plaques, ensuite encrées puis pressées, fassent apparaître à l'endroit la partition sur le papier. Elle permettait d'améliorer, outre l'aspect esthétique, la lecture et surtout le lien des symboles. Son implantation en France fut plus tardive qu'au Nord de l'Europe, en raison du monopole des Ballard.

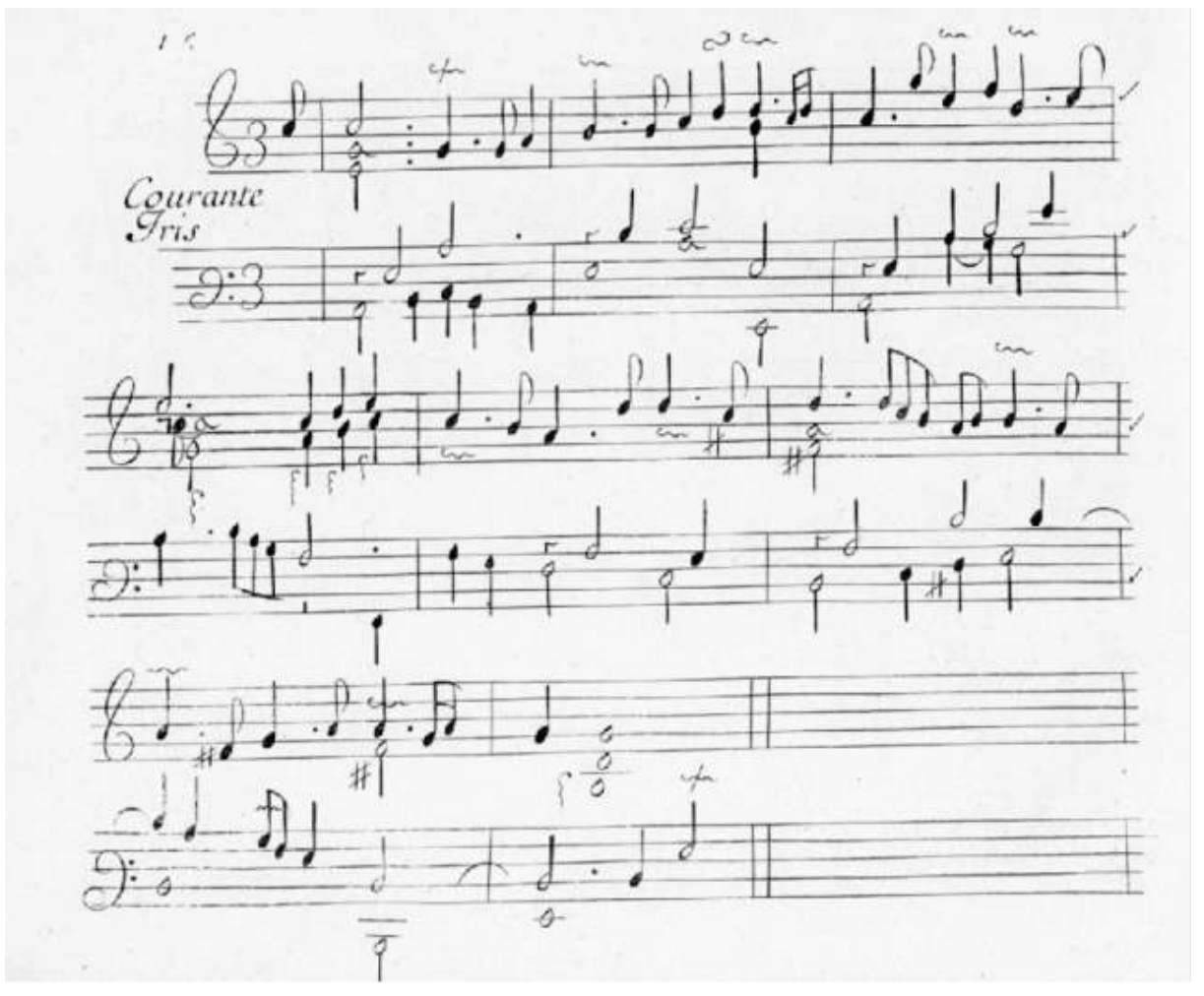




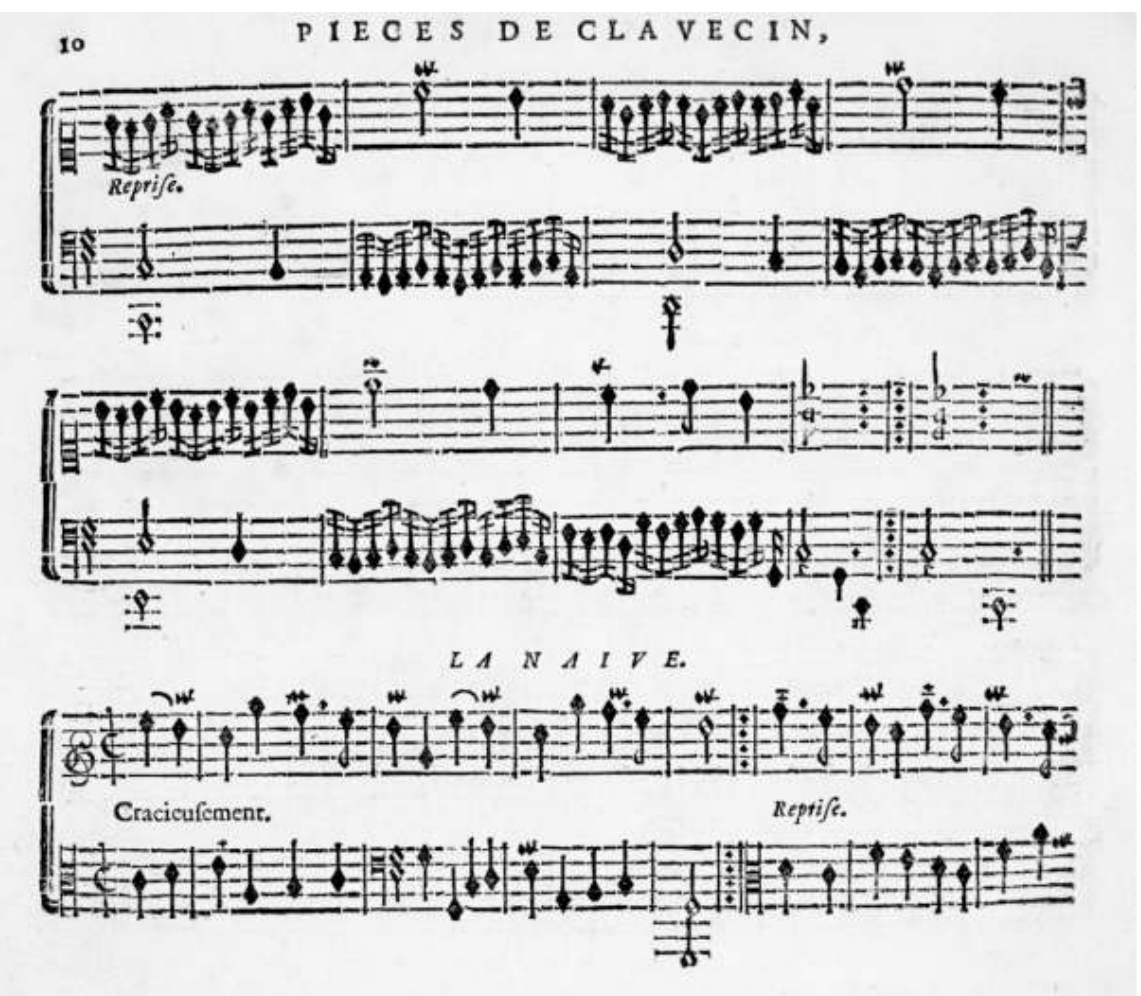

Ex. 1 : Comparaison de la gravure avec la typographie. Deux pièces de clavecin : la courante Iris de Chambonnières gravée par Jollain en 1670 et la Naïve de Durocher imprimée par Ballard en 1733

Heureusement, la gravure, considérée comme un autre mode de diffusion de la musique que celui de l'impression par typographie, fut un moyen efficace d'instituer une édition concurrente à celle des Ballard et, par voie de conséquence, de contrer leur privilège. C'est seulement en 1660 que la première gravure réalisée en France voit le jour avec 500 exemplaires tirés des Fugues et Caprices à quatre parties mises en partition par François Roberday ${ }^{6}$. Vers 1680, un timide concurrent à la famille Ballard apparait, sous forme de musique gravée, et à partir de 1690 avec l'ouverture de la boutique d'Henry Foucault, « À la Règle d'or ${ }^{7}$ ». Il devient l'un des grands marchands de musique de l'époque, prédécesseur de Boivin dans la boutique de la rue Saint-Honoré portant l'enseigne de la Règle d'or.

5 C'est avec Jollain, en 1670, que Chambonnières publie le premier livre de pièces de clavecin de l'histoire française (Cf. Ex. 1). Il ouvre, au sein de l'école de clavecin, la voie à une nouvelle pratique qui va se développer et se multiplier au début du $\mathrm{xVIII}^{\mathrm{e}}$ siècle, passant ainsi d'une transmission orale à une transmission éditoriale qui engendre un contrôle musical de l'œuvre. L'écriture pour clavecin sera modifiée, et de plus forte manière, l'écriture ornementale 8 .

6 Mais est-ce uniquement grâce aux avancées techniques et au contournement du monopole des Ballard que Chambonnières a pu éditer le premier livre de pièces de clavecin de l'histoire française? 


\section{L'édition de pièces de clavecin et les finances des clavecinistes}

7 Les pièces pour clavecin de Chambonnières circulent dans le milieu musical depuis plusieurs décennies grâce aux manuscrits9. En 1670, dans sa préface, le compositeur et claveciniste explique la raison de la publication tardive de ses pièces. Seule l'existence de copies médiocres qui déforment ses œuvres et circulent en Europe l'a décidé à imprimer :

Cependant, les avis que je reçois de différens lieux qu'il s'en fait un espèce de commerce presque dans toutes les villes du monde, où l'on a la connoissance $d u$ clavecin, par les copies que l'on en distribue quoy qu'avec beaucoup de deffauts et ainsi fort à mon préjudice, m'ont fait croire que je devois donner volontairement ce que l'on m'otoit avec violence et que je devois mettre au jour moy-même ce que d'autres y avoient déjà mis à demy pour moy; puis qu'aussi bien les donnant avec tous leurs agréments comme je fais en ce recueil, elles seront sans doute et plus utiles au public et plus honorables pour moy que toutes ces copies infidèles qui paroissent sous mon nom ${ }^{10}$.

Chambonnières, âgé de 69 ans, en fin de carrière, se cache bien de donner une autre raison de cette publication, raison qui pourrait être gênante et nuirait à sa réputation. Le claveciniste omet de mentionner ses problèmes financiers qui le poussent à vouloir récupérer de l'argent par tous les moyens. En effet, depuis quelques années, il accumule les pertes pécuniaires. En 1652, l'armée de la Fronde dévaste la région de Brie et le domaine hérité de sa grand-mère maternelle. En 1657, sa deuxième femme obtient le divorce et lors de la séparation de biens prononcée par le Parc civil du Châtelet de Paris, elle est autorisée à vendre la terre et la métairie du Petit-Plesse-en-Brie. De plus, peu à peu, Chambonnières, pour des raisons précises inconnues, se voit disgracié de la cour du roi. Les musicologues émettent plusieurs hypothèses sur les causes de cette disgrâce, dont la première serait liée au titre de noblesse ajouté à son nom :

D'autre part, en cette année 1662 qui vit Jean de La Fontaine être condamné pour usurpation de titres de noblesse, la disgrâce de Chambonnières ne s'explique-t-elle pas aussi par ses prétentions nobiliaires («baron» ou «marquis» suivant les textes) avec lesquelles Louis XIV ne plaisantait guère ${ }^{11}$ ?

Durant toute sa vie, Chambonnières s'était constamment préoccupé d'améliorer sa position sociale, en commençant par prendre le nom de la propriété de son grandpère maternel, puis en prenant le titre de baron, et pour finir en se désignant comme marquis. Louis XIV n'aimait guère que l'on se pare de titres sans permission et ceci a pu causer la perte de la position de Chambonnières comme joueur d'épinette ${ }^{12}$.

Pourtant, un autre claveciniste du roi, d'Anglebert, selon les recherches effectuées par le musicologue Kocevar au travers d'archives ${ }^{13}$, aurait lui aussi ennobli son nom, issu d'un surnom, en amenant progressivement le « $d$ » vers une minuscule et en ajoutant un tiret entre son prénom et son nom Jean-Henry, sans être pour autant disgracié (dit Anglebert n'est qu'un surnom emprunté très tôt dans la famille pour la distinguer de nombreuses familles vivant sous le nom de Henry aux alentours de Bar-le-Duc) ${ }^{14}$.

10 La deuxième raison de la disgrâce de Chambonnières serait son " caractère impossible $e^{15}$ " qui le pousserait à refuser de jouer la basse continue. Cette rumeur, encore considérée aujourd'hui par les musicologues, est lancée par le violiste Jean Rousseau en 1688, non pas pour des raisons de caractère, mais pour des raisons d'incapacité : « M. de Chambonnières ne sçavoit pas accompagner, \& que ce fut pour ce sujet qu'il fut obligé de se défaire de la 
charge qu'il avoit chez le Roy, \& de s'en accomoder avec Monsieur d'Anglebert ${ }^{16}$. » Cela est difficilement concevable de la part du grand claveciniste de ce siècle. Dès 1641, Chambonnières avait mis en place sa série de concerts payants de l'« Assemblée des honneste curieux », il y participait comme claveciniste et continuiste. Mais le continuo ne devait pas être sa pratique préférée. Chambonnières a pu refuser de jouer à cette place. En 1992, le claveciniste Sempé explique que ce poste de continuiste ne correspondait pas au rôle que le grand claveciniste du roi s'attribuait : «Chambonnières, donc, a refusé la tradition de Lully, ceci expliquant son éloignement de la Cour, très vraisemblablement pour avoir refusé de faire partie de la basse continue de la machine orchestrale de Lully ${ }^{17}$ . » La disgrâce aurait commencé dès 1657. Louis XIV, avec qui il avait pourtant dansé quelques années auparavant dans le Ballet royal de la Nuit de Lully, choisit Étienne Richard, organiste de Saint-Jacques-de-la-Boucherie comme maître de clavecin. L'ami mathématicien de Chambonnières, Christian Huygens, laisse entendre que sa dégradation semble de surcroît toucher son légendaire talent (ce qui a pu alimenter les écrits de Jean Rousseau ${ }^{18}$. De cette période de précarité financière, datent sûrement les deux anecdotes souvent citées à propos du carrosse et des chevaux du claveciniste déchu :

Chambonnière qui jouoit si bien du Clavecin, \& qui savoit parfaitement la Musique, étoit un homme fort agréable et bien fait de sa personne ; mais il étoit d'une vanité insupportable; et ne se contentant pas de se faire gentilhomme, il vouloit encore faire le grand Seigneur. Il avoit un carrose traîné par deux méchants chevaux, avec un page en effigie et rempli de foin attaché sur le derrière. Étant au Cours avec ce carrosse, où les carrosses se suivent en marchant lentement suivant la coutume, les chevaux du carrosse qui suivoient le sien, sentant le foin devant eux, se mirent à prendre le page par les jambes. Quelqu'un, qui s'en aperçut, cria au cocher : Prenez garde à vos chevaux, ils mangent le page de Monsieur ${ }^{19}$.

À propos de chevaux, je ne sçaurois que je ne mette icy la pitoyable aventure des chevaux de Chambonnière, cet excellent joüeur de clavessin. Il avoit un carrosse, mais, faute de nourriture, il envoyait paistre ses chevaux sur le rempart du Marais. Je vous laisse à penser en quel estat ils etoient. Des escorcheurs les prirent pour des chevaux [condamnés], et un beau matin ils les escorchèrent tous les deux ${ }^{20}$.

11 En 1660, on propose le poste de claveciniste de la chambre du roi de Chambonnières, à Louis Couperin qui refuse par respect pour son ancien professeur. En 1662, Chambonnières est privé de se rente de 1000 écus. L'homme d'État et poète Constantin Huyghens tente alors, mais sans succès, de recommander le musicien auprès de l'Électeur de Brandebourg le 31 août 1662 en écrivant au baron de Schwerin ${ }^{21}$. La même année, ayant besoin d'argent, Chambonnières vend 2000 livres la survivance de sa charge à JeanHenry d'Anglebert.

$12 \mathrm{Au}$ vu de tous ces faits, nous pouvons supposer que l'édition du premier livre de pièces de clavecin de l'histoire de l'école française a pu être motivée par des raisons financières. La naissance de l'édition des pièces de clavecin a donc une origine peu reluisante. Qu'en estil des compositeurs suivants?

13 D'autres évènements personnels financiers vont guider le paysage éditorial pour clavecin. A contrario de Chambonnières, lorsque d'Anglebert édite, il est au sommet de sa gloire et installé dans le système de la cour $^{22}$. En 1689, il est claveciniste du roi, depuis 17 ans officiellement, mais tient cette position depuis presque 30 ans. Âgé de 70 ans, il nous livre avec fierté l'œuvre de toute une carrière. L'ouvrage est donc conséquent, il contient une des tables des agréments les plus célèbres de l'histoire du clavecin car très soignée et précise, ainsi que des pièces de clavecin, des arrangements d'œuvres de Lully ou de pièces de luth, des fugues pour orgue et les Principes de l'Accompagnement. Le parcours d'un 
troisième claveciniste, Louis Marchand, ressemble bien plus à celui de Chambonnières. Marchand publie en 1699 son premier livre de clavecin. Il est alors marié, depuis le 5 février 1689, à Marie-Angélique Denis, fille de Louis Denis facteur d'instruments, organiste de Saint-Barthélemy. Titulaire d'un certain nombre de postes d'organistes à Paris (ceux des Cordeliers, des Jésuites de la rue de Saint-Jacques et de Saint-Benoit), il court sans cesse après plusieurs tribunes, dont celle de Saint-Merry que le Mercure Galant de novembre 1686 qualifie d'estimable : « Les orgues de cette église qui ont la réputation d'être aussi bonnes que l'organiste est habile ${ }^{23}$. " Le graveur de son premier livre, Roussel, annonce que Marchand publiera très régulièrement ses compositions : « L'auteur donnera au public tous les trois mois, une suitte de pièces de clavecin alternativement avec une suitte de pièce d'orgue de chaque ton ${ }^{24}$." Pourtant, contrairement à l'annonce, le deuxième livre de Marchand de 1700-1701 sera gravé par un concurrent : Baussen ${ }^{25}$ et les publications s'arrêteront par la suite. L'arrêt subit de la liste de ses publications peut s'expliquer par les démêlés judiciaires de Marchand avec sa femme, démêlés qui commencent en 1701. Les époux vivaient en désaccord depuis longtemps, mais les choses s'enveniment alors à un point tel que la rupture est inévitable. Pour certains musicologues, comme Denis Morrier en 2006, Marchand décida de réduire ses revenus afin de ne pas les verser à son ancienne femme :

L'épouse obtint en 1702 la séparation des biens et d'habitation d'avec son mari, dont elle devait continuer à percevoir la moitié des revenus. Dès lors, Marchand fit en sorte de ne pas gagner trop facilement un argent dont il n'aurait pas eu la jouissance intégrale : ainsi joua-t-il gratuitement aux Cordeliers jusqu'à sa mort et abandonna-t-il la publication de ses œuvres, qu'il avait commencée en $1699^{26}$.

14 Ainsi, les finances personnelles des compositeurs modulent le paysage éditorial pour clavecin. Non seulement la situation pécuniaire influence l'apparition des éditions de pièces de clavecin, mais nous allons constater qu'elle modèle aussi la forme et le fond de chaque recueil.

\section{Une forme éditoriale propice à la vente}

15 Avec la gravure, les musiciens publient à compte d'auteur - pour autant qu'ils aient des ressources pécuniaires suffisantes, généralement obtenues par la bonne grâce de la riche noblesse -, et en s'associant aux graveurs et revendeurs, pour faire face aux frais. Ainsi, un jeune musicien sans mécène ne pourra pas éditer un recueil de la même valeur qu'un de ses aïeux plus expérimenté et mieux entouré. Les dédicaces écrites au début des livres de pièces de clavecin permettent d'identifier ces mécènes. Si Dandrieu publie en 1704 un recueil fort onéreux, c'est sûrement grâce à l'appui de son dédicataire : le Chevalier de Septueil. "Monsieur Robert, chevalier, seigneur de Spteuil, Conseiller du Roy en ses Conseils et Président en sa Chambre des Comptes ${ }^{27}$ » écrit-il dans sa dédicace. En effet, il est très étonnant de constater le luxe de cette première édition de jeunesse pour Dandrieu qui est alors âgé de 22 ans et ne vit que de la pratique de l'enseignement et de ses concerts au sein du cercle fermé du Palais-Royal chez les Orléans. Cette première édition est imprimée entre le moment où Dandrieu prend ses fonctions à Saint-Merry, le 4 janvier 1704 et sa nomination officielle à ce poste, le 19 juillet 1705 . La dédicace de Dandrieu est placée dans le médaillon du frontispice : les armes de Septueil, personnage important - un taureau avec une étoile entre les cornes - figurent sur la gravure. Une seconde dédicace dédiée au même, tenant sur une page avant le frontispice, est plus développée. Dandrieu évoque l'anecdote selon laquelle le Chevalier de Septeuil le 
considérerait comme le légitime héritier de son aîné Lebègue, mort en 1702. Le poste de Lebègue à l'orgue de Saint-Merry, est occupé à sa mort par Mayeux. Mais ce dernier est malade en 1704 et il semble que ce soit Monsieur de Septeuil qui ait proposé Dandrieu pour reprendre la place laissée vacante par Lebègue, puis Mayeux. En 1974, la musicologue Brigitte François-Sappey écrit que la partition de Dandrieu de 5 livres est une «édition de luxe sur beau papier avec frontispice provenant d'artistes connus [Desmarest] ${ }^{28} »$, ce qui explique son prix élevé. L'indication du prix ne se trouve pas sur le recueil lui-même, mais dans les catalogues. La seconde édition de ce premier livre de jeunesse de Dandrieu entre 1715-1720, d'une valeur de 40 sols était donc beaucoup moins prestigieuse et chère, avec un frontispice d'un graveur inconnu, donc sûrement peu célèbre et un papier plus fin.

En 1706, lorsque Rameau publie son premier livre de clavecin, il n'est encore qu'un jeune provincial tout juste arrivé à Paris $^{29}$. Il n'écrit aucune dédicace et montre ainsi, comme Le Roux en $1705^{30}$, qu'il n'a pas de mécène. Son frontispice richement orné d'instruments est identique à celui de Dandrieu de 1704. Les deux jeunes compositeurs ont eu le même graveur et la même page de garde dont seul le contenu de la cartouche change ainsi que celui du médaillon ${ }^{31}$. Le graveur est Roussel, le dessin est de Desmarest. Il représente tous les instruments : clavecin, théorbe, luth, chalumeau, cornemuse, cor, triangle, trompette, serpent, échelette, lyre, viole, violon, bombarde, orgue positif, harpe, tambour, guitare, timbales, violone, trompette marine, tambour de basque. Ce dessin richement fourni sera réutilisé dans d'autres éditions puisqu'il faisait partie des fonds de l'imprimeur Foucault ${ }^{32}$. Ceci nous indique que Rameau (et non Dandrieu qui semble être le premier à bénéficier de cette gravure?) n'avait pas les moyens d'acheter une gravure originale pour son livre, mais a pu bénéficier d'un système de partage moins onéreux.

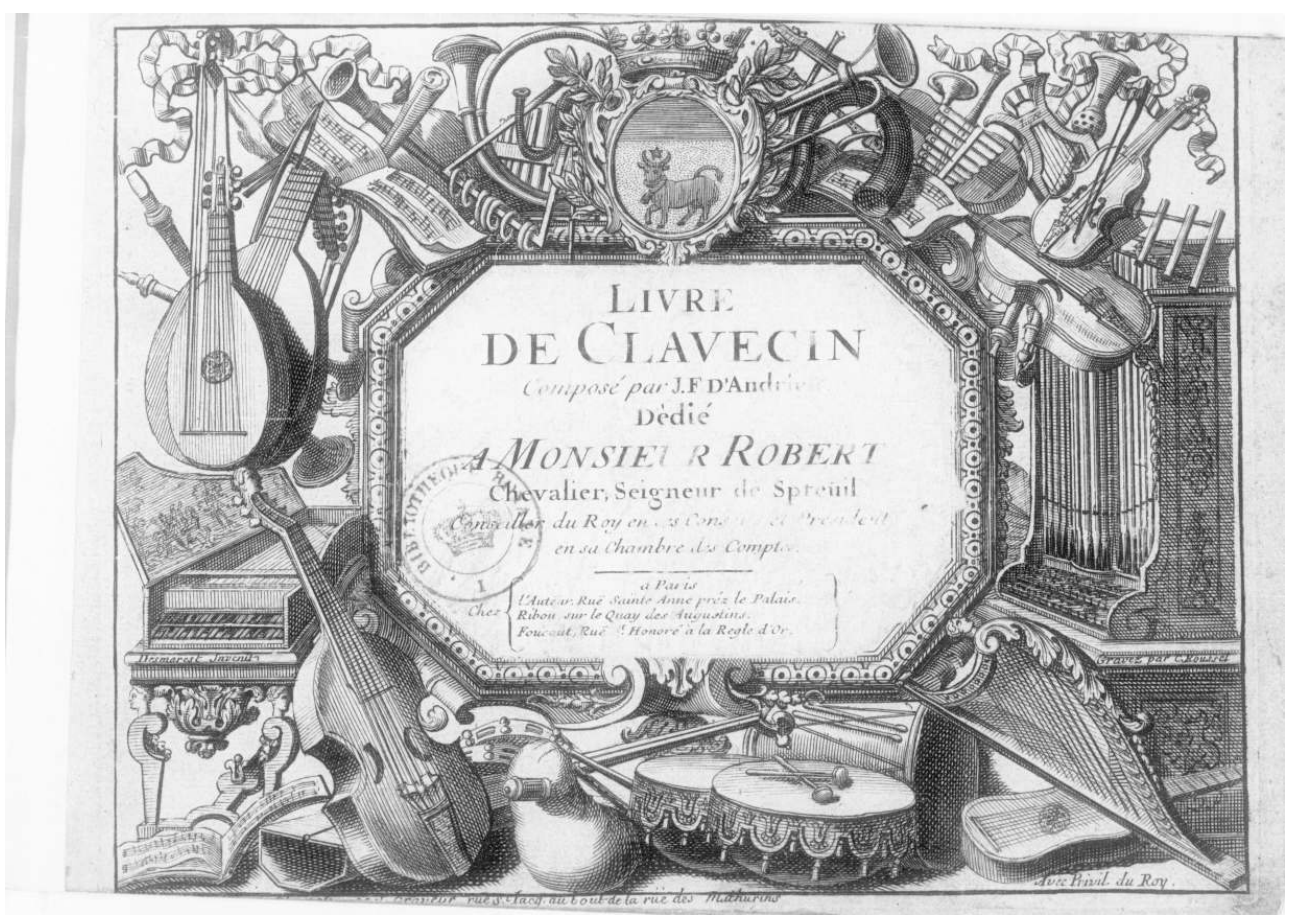




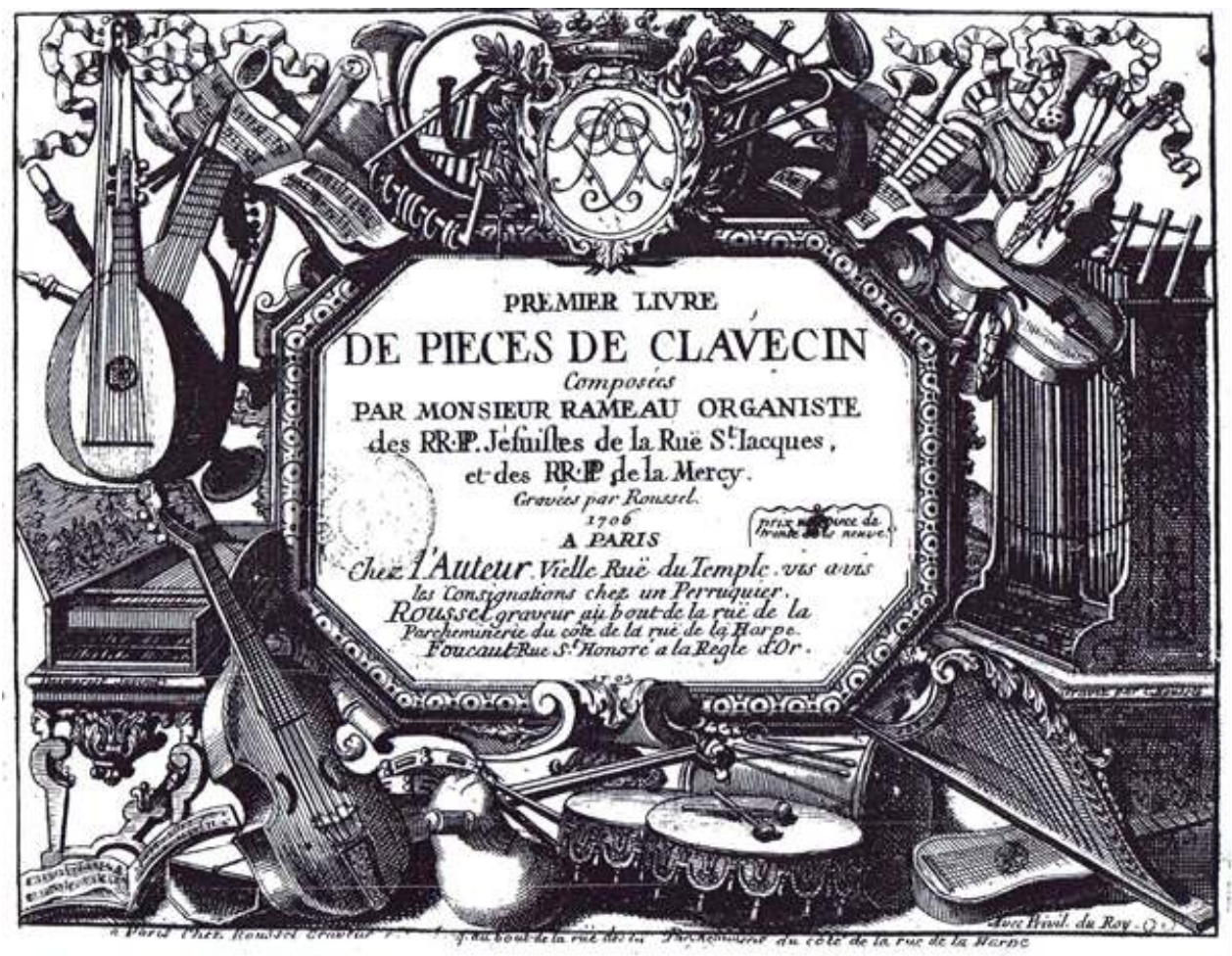

Ex. 2 : Frontispice du premier livre de Dandrieu de 1704 et du premier livre de Rameau de 1706

17 Malgré la finesse de ces pages de garde, l'allégorie de la musique présente dans certains exemplaires du livre de d'Anglebert reste la gravure la plus subtile et la plus étoffée de l'ensemble des recueils de pièces de clavecin. Encore une fois, ce livre est le plus pécuniairement démonstratif. L'allégorie entame l'édition de d'Anglebert, avant un portrait du compositeur et avant le frontispice lui-même. Le dessin est de Mignard, peintre du roi et la gravure de Vermeulen comme pour le portrait. À quelques variantes près, Mignard semble s'être inspiré des descriptions de l'érudit italien Cesare Ripa dans son Iconologie, recueil d'emblèmes et de figures destiné aux poètes et aux peintres. La musique y est décrite en ces termes :

C'est une Femme qui regarde fixement un Livre ouvert, qu'elle tient d'une main, \& une plume de l'autre, pour corriger sa tablature ; ayant pour cet effet à ses pieds un Luth, une viole, \& des Flustes, pour accorder l'harmonie à celle de sa voix. [...] A cette dernière Figure peut estre joint fort à propos celle d'une femme qui joüe d'un Cistre, où se void une Cigale à la place d'une Corde qui est rompüe : outre qu'elle se fait remarquer par un Rossignol qu'elle a sur la teste, par une grand Vase, qui est à ses pieds, \& par une lyre avec son $\operatorname{archet}^{33}$.

De ces divers éléments iconologiques, Mignard retient l'image d'une jeune fille assise sur un globe d'azur qui, selon Ripa, signifie que toute l'Harmonie sensible se fonde sur l'Harmonie des Cieux, que les Pythagoriciens ont connue. Elle lit un fragment musical portant l'inscription " DIVUM HOMINUMQVE VoluPTAS " (plaisir des dieux et de l'homme) et tient une lyre dont elle joue. Ripa précise que " par le Livre de Musique est enseigné la reigle qu'il fait tenir, pour apprendre par la veuë ce merveilleux Art à ceux qui ne le savent pas $^{34}$.» Sa tête est surmontée d'un rossignol, symbole de la "merveilleuse mélodie. » Un concert d'angelots accompagne la muse, qui est entourée à droite d'un cabinet d'orgue, et à gauche, par un clavecin à deux claviers sur un piètement torsadé et retourné. Un livre de musique oblong - comme les Pièces de clavecin de d'Anglebert - est 
posé sur un pupitre sur le couvercle fermé de l'instrument. Pour la première fois, le clavecin est représenté avec son piètement torsadé et tourné (précédemment, dans les recueils de Chambonnières et Lebègue, le clavecin est représenté sans piètement).
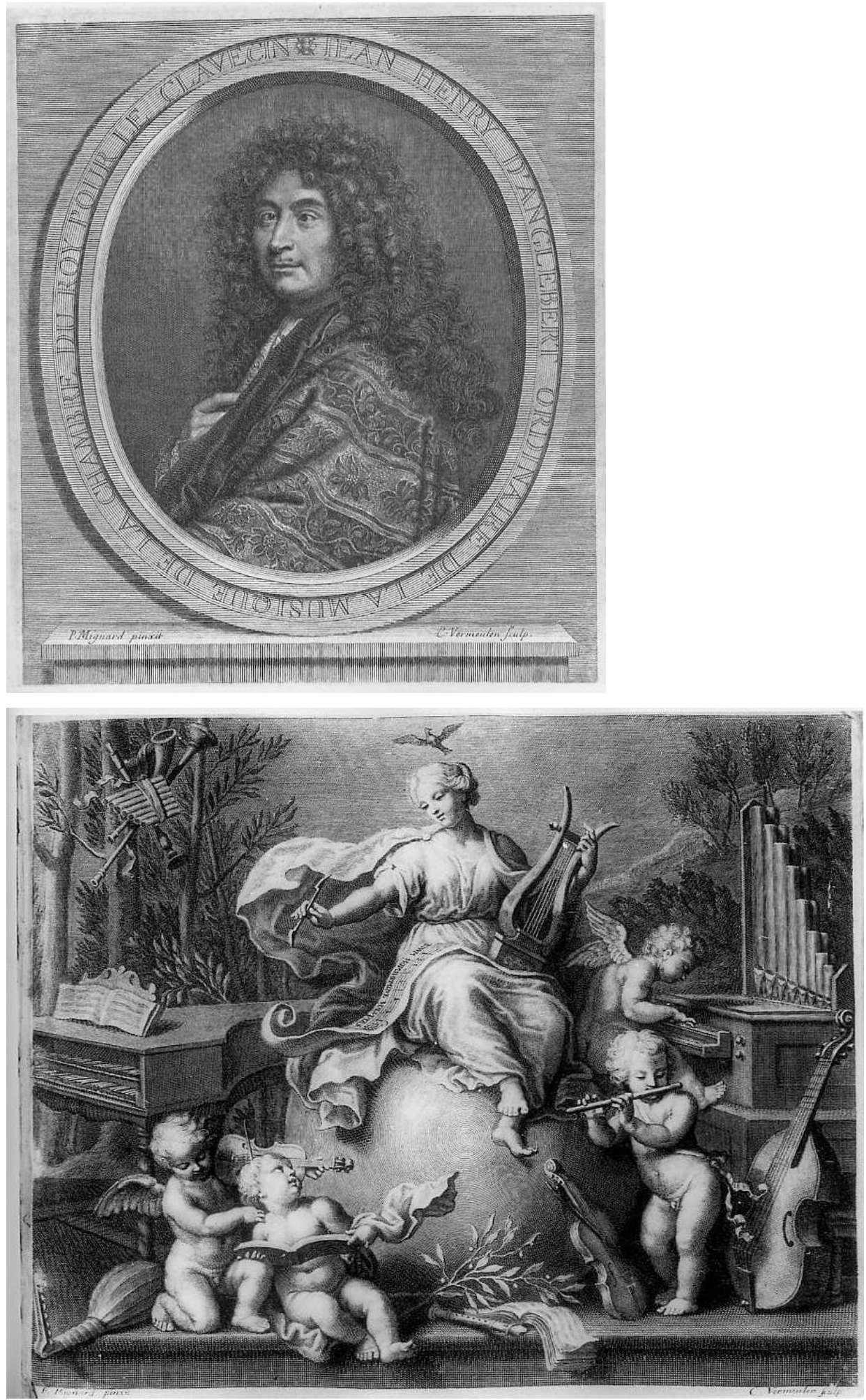

Ex. 3 : Portrait de d'Anglebert et allégorie de la musique gravés dans l'édition de 1689

19 Les symboles choisis répondent à des codes précis issus d'un langage connu et commun de l'époque. D'Anglebert est le seul claveciniste à insérer son portrait dans un recueil de 
pièces. Mais, ainsi, le prix n'était pas accessible à tous. La valeur du recueil augmentait avec cette gravure et le portrait qui suivait, d'où leur présence dans tous les tirages, mais non dans tous les recueils retrouvés. Avec ces deux formes de recueils, d'Anglebert élargit son panel d'acheteurs. Car si les clavecinistes publient, c'est pour vendre. Quelles stratégies mettent-ils en place avec les commerciaux pour vendre leurs œuvres?

Dès le quatrième recueil de l'histoire du clavecin en France, des innovations commerciales apparaissent. Ces nouveautés touchent la forme même de la musique. En 1689, d'Anglebert insère à la suite traditionnelle des pièces de clavecin, 15 transcriptions tirées d'opéras de Lully, car « Il faut avoüer que les ouvrages de cet homme incomparable [Lully] sont d'un goût fort supérieur a tout autre » s'explique-t-il dans sa préface. En 1701, Dieupart propose avec ses pièces de clavecin, une version « mise en concert par Monsieur Dieupart pour un violon \& flûte avec une Basse de Viole \& un Archilut ${ }^{35}$.» En 1705, Le Roux écrit pour le même effectif et ajoute une troisième version pour deux clavecins ${ }^{36}$. Enfin, en 1707, Élisabeth Jacquet de La Guerre propose une édition, dont aujourd'hui personne ne semble comprendre le format exact, intitulée : Pièces de Clavecin qui peuvent se joüer sur le viollon ${ }^{37}$.

21 Au-delà de ces innovations musicales attractives, l'édition doit être une source de pratique pour les acheteurs. Le format oblong, utilisé pour les gravures parisiennes de ce tournant de siècle permet un usage facile et aisé. En 1761, le membre de l'Académie des Sciences, François-Alexandre-Pierre de Garsault écrit, pour définir le clavecin, que « c'est l'instrument des Demoiselles à marier \& des Dames $^{38}$.» La pratique intime des salons nécessite un ouvrage facilement manipulable. Les clavecinistes doivent vendre leurs livres à ce cercle issu des salons, qui inclut souvent leurs élèves. Ils ouvrent la voie à une technique éditoriale commerciale ${ }^{39}$. Mais les tirages sont limités et le prix élevé par rapport aux produits de la vie courante, car les procédés de gravure sont coûteux: planches d'étain et de cuivres poinçonnées. La comparaison des prix affichés des partitions nous permet de mettre en place une grille de valeurs :

\begin{tabular}{|l|l|}
\hline Compositeurs & Prix \\
\hline Chambonnières 1 & 10 livres en 1734 \\
\hline Chambonnières 2 & 10 livres en 1734 \\
\hline Lebègue 1 & 20 livres en 1734 \\
\hline Lebègue 2 & 20 livres en 1734 \\
\hline Jacquet de La Guerre 1 & $?$ \\
\hline D'Anglebert & 10 ou 12 livres en 1689 \\
\hline Marchand 1 & 2 ou 3 livres en 1742 \\
\hline Marchand 2 & 2 ou 3 livres en 1742 \\
\hline Clérambault & 25 sols (3 livres en 1734) \\
\hline Dandrieu & 5 livres en 1704 \\
\hline
\end{tabular}




\begin{tabular}{|l|l|}
\hline Le Roux & 10 ou 11 livres 20 en 1705 \\
\hline Rameau & 30 sols en 1706 \\
\hline Jacquet de La Guerre 2 & 3 livres et 10 sols en 1707 \\
\hline
\end{tabular}

Ex. 4 : Tableau comparatif du prix des recueils de pièces de clavecin de 1670 à $1707^{40}$ Dix livres ${ }^{41}$ semble être le prix le plus commun pour les pièces de clavecin au tournant du XVII ${ }^{\mathrm{e}}$ siècle. On le retrouve chez d'Anglebert et Le Roux (ou même Couperin plus tard en 1713). Le recueil de 1707 de Jacquet de La Guerre coûte 3 livres et 10 sols. Une livre correspondant à 20 sols, ainsi le premier recueil de Rameau de 30 sols équivaut à 1,5 livres. En 1964, selon le musicologue Thurston Dart, le recueil de Clérambault était vendu 25 louis $^{42}$, or le louis d'or était très fluctuant, à quoi correspondrait cette somme ? D'une valeur de 10 livres en 1640, le louis passa par une valeur de 20 livres en 1709 et continua à fluctuer. En comparaison avec les autres recueils, ce prix parait exorbitant pour un si petit recueil (l'édition contient deux suites, soit 16 pièces). Ne serait-ce pas plutôt 25 sols ? Ceci correspondrait à environ 1,25 livres, ce qui se rapprocherait plus des autres recueils de l'époque notamment de celui de Rameau qui a sensiblement le même format. Le prix varie aussi si les feuilles sont reliées ou non, ainsi dans le recueil de Le Roux, nous pouvons lire ajouté à la main sous le prix initial gravé de " 10 lt en blanc » ce qui signifie non relié, un texte manuscrit: «relié 11 lt 20 ». Pour l'œuvre de Chambonnières, composée de 61 pièces, la séparation en deux volumes, choisie sûrement par le graveur Jollain, permet de vendre à moindre prix et donc de vendre plus ${ }^{43}$. Dans son livre de 1976, la musicologue Anik Devriès compare les prix des recueils uniquement entre 1734 et 1742, notant une augmentation du coût de la musique durant cette période. Chaque livre de clavecin de Marchand est vendu 2 livres par Boivin et 3 livres par Leclerc ${ }^{44}$. Avant cette date nous ne connaissons pas le prix des recueils de Marchand. En 1734 dans le catalogue du commerçant Leclerc, le livre d'Anglebert est au même prix qu'en $1689: 10$ livres $^{45}$. Ainsi, ces catalogues tardifs peuvent nous donner une idée réelle du prix du recueil lors de sa sortie. En 1734, chaque livre de Chambonnières est vendu 10 livres (prix le plus courant des recueils de pièces de clavecin de notre période étudiée) ${ }^{46}$, chaque livre de Lebègue est vendu 20 livres (rappelons que Lebègue a écrit deux recueils imposants avec un total de cent-deux pièces) ${ }^{47}$, et le recueil de Clérambault coûte 3 livres (le prix semble avoir doublé depuis sa sortie) ${ }^{48}$. Grâce à sa notoriété de plus en plus importante dans les années 1730 (et une augmentation générale des prix), le premier livre de Rameau passe de 30 sols en 1706 à 7 livres en $1734^{49}$. Ce recueil est apparemment devenu rare puisqu'il est plus cher que les livres suivants du compositeur qui sont bien plus fournis (6 livres et 2 livres). D'après le musicologue John Walter Hill, la raison commerciale de ces éditions ne fait aucun doute :

À partir de la fin des années 1680, un nombre important de compositeurs français de musique pour clavecin n'avaient pas de nomination à la cour royale [...] Même lorsqu'un compositeur était nommé à la cour, sa musique pour clavecin était désormais imprimée pour des amateurs fortunés et éduqués, plutôt que d’être préservée seulement sous forme de manuscrit ${ }^{50}$.

En général, ces prix nous indiquent que le public des pièces de clavecin faisait partie d'une population aisée. Ceci ne contredit en rien la portée pédagogique de ces recueils. 
Quel était le niveau musical de ce public? Les clavecinistes adaptent-ils leur écriture afin de brasser une plus large population?

Clérambault, ou peut-être son graveur Roussel, propose en fin du premier livre, la possibilité de commander des pièces sur mesure : «CEUX qui souhaiteront des pièces particulières, / faciles, ou d'exécution à la moderne, n'auront / qu'à s'adresser à lui ${ }^{51}$. » Cette annonce, tout en étant commerciale, dénote d'une volonté de s'adapter à un public varié. Elle tendrait à classer les pièces proposées par Clérambault dans son premier livre comme des pièces peu faciles et non modernes. Selon le musicologue Vincent Bernhardt, la précision des ornements dans les partitions du début du XVIII ${ }^{\mathrm{e}}$ siècle est une nécessité pédagogique qui n'avait pas lieu d'être précédemment lorsque la musique s'adressait plus généralement à des musiciens avertis :

En général, les personnes à qui étaient destinées ces partitions notées avec une certaine simplicité (certes toute relative) étaient des musiciens suffisamment compétents pour être en mesure d'en donner immédiatement une interprétation convenable, exécutée dans le style appropriée - et le compositeur n'avait pas à se donner la peine lui-même d'ajouter tous les détails de l'exécution dans sa musique (l'ornementation étant l'essentiel de ces " particularités pratiques»). Si une copie ornée était nécessaire, que ce soit pour une raison pédagogique (enseignement à un élève ayant besoin d'apprendre le style ornemental alors en vogue), pratique (partition étant destinée à être jouée par un amateur peu au courant des vrais raffinements de l'exécution - souvent connus des seuls professionnels), ou quelle qu'elle soit, ce n'était certes pas au créateur de se charger de la réaliser, mais plutôt à ses proches ou à ses élèves. [...] Il y faut distinguer à la fois un critère de destinataire (car on n'écrit pas de la même façon lorsqu'on destine sa partition à soi-même, à ses proches ou à sa famille, à un noble protecteur, à des débutants, à des musiciens confirmés, ou à des amateurs), et un critère d'époque ${ }^{52}$.

L'écriture simplifiée, notée par Davitt Moroney dans les pièces de Chambonnières, confirme ce point de vue :

Les corrections qu'il apportait à ses propres œuvres peuvent être étudiées en comparant les versions manuscrites aux dernières versions qu'il a publiées en 1670 ; pour lui, le fait de corriger consistait presque toujours à simplifier, à réduire toute confusion afin de produire un style raffiné et épuré [...] (Certains experts s'étonnent même qu'il publie ses morceaux " moins intéressants ", mais c'est confondre complexité et intérêt) ${ }^{53}$.

Ainsi, un certain nombre de clavecinistes va ajouter une table explicative des agréments dans son recueil afin de guider et rassurer les musiciens amateurs. Chambonnières, Lebègue, d'Anglebert, Dieupart, Le Roux et Rameau, soit 6 compositeurs sur 9, vont créer leur propre table. Celle-ci, succincte au début de l'histoire éditoriale, se complexifie au fur et à mesure. Est-ce, de la part de compositeurs, une correction des insuffisances des tables précédentes?



Ex. 5 : Table de Chambonnières de 1670 




Ex. 6 : Table de Le Roux de 1705

27 La préface de Lebègue, du premier recueil imprimé de 1677, indique la difficulté de l'auteur à livrer des préludes non mesurés à un public éventuellement non averti : «La grande difficulté de rendre cette metode de Preluder assé intelligible a un chacun ${ }^{54}$. » En effet, contrairement à Louis Couperin dont les préludes manuscrits étaient notés uniquement en rondes, Lebègue adopte une notation mixte, mêlant à ces rondes certains rythmes qui, même s'ils ne doivent pas s'interpréter littéralement, facilitent la compréhension et l'interprétation harmonique et mélodique de ces mystérieuses pièces. Ce choix d'une écriture mixte par Lebègue peut être pensé comme une tentative de lisibilité auprès d'amateurs. Malheureusement, aucun de ses préludes n'est présent sous forme manuscrite, nous ne pouvons pas apporter de preuves à cette supposition. Il faut croire que ces essais de notation n'ont pas été suffisants pour les musiciens étrangers, car, lors de la deuxième édition du premier livre de Lebègue chez Roger à Amsterdam, la page de titre contient le commentaire suivant: "Comme plusieurs Personnes ignorent la Manière de joüer ces Pieces et Par Consequent n'en Connoissent pas la Beauté on Avertie ceux qui seront curieux de les Entendre de S'Adresser a Monsieur Marquis Maistre de Clavessin a Amsterdam ${ }^{55}$. » Ajouté à cela la demande d'explication sur l'interprétation des préludes non mesurés par un amateur anglais en $1684^{56}$, Lebègue supprimera cette forme de pièces de son deuxième livre. D'autres différences de pratiques éditoriales entre le premier livre de 1677 et le second de 1687 du claveciniste, indiquent qu'il a pris en compte les difficultés rencontrées par le public. Dans son second livre, Lebègue simplifie l'écriture musicale afin de limiter les ambiguïtés ornementales et les corrections futures probables. En novembre 1687, le Mercure Galant avisait le public de la parution de ce nouveau recueil et précisait : « vos amies seront peut-être bien aises de l'apprendre, parce qu'il les envoye dans toutes les villes du royaume, où elles ne sont pas moins estimées par la facilité qu'il y a de les apprendre, qu'à cause de leur extrême beauté. » En conséquence, Lebègue passe de $14,74 \%$ d'agréments dans le premier livre à $10,31 \%$ dans le second ${ }^{57}$. Nous constatons que pour chaque deuxième livre de pièces de clavecin, le compositeur a écrit moins d'agréments, comme s'il voulait adapter son écriture :

- Lebègue : de $14,74 \%$ à $10,31 \%{ }^{58}$

- Jacquet de La Guerre : 17,60 \% ${ }^{59}$ à 15,61 \%

- Marchand : de 13,82 \% à 13,09\% ${ }^{60}$

- Rameau : de $13,33 \%$ à $10,33 \%{ }^{61}$.

De plus, l'écriture de Lebègue pour le second livre est mieux contrôlée dès le premier tirage. En effet, le premier livre compte 6 tirages avec beaucoup de changements 
d'ornements, alors que le second, n'a que 2 tirages retrouvés avec une seule modification ornée apportée.

Chez Dandrieu, rappelons-le, la première édition de 1704 est luxueuse, mais très peu d'agréments sont notés. Le public concerné serait celui d'amateurs riches non virtuoses, car les pièces sont assez faciles, sûrement des élèves auprès desquels Dandrieu enseignait oralement les agréments, avec la partition sous les yeux. L'aspect pédagogique ressort plus dans sa seconde édition, vers 1715 : elle est moins onéreuse et enrichie d'un grand nombre d'agréments ajoutés et notés. Les taux d'agréments basculent de 9,48\% dans la première édition (moyenne la plus basse de tous les recueils de l'époque) à 13,47 \% pour la deuxième édition. Dans cette deuxième édition, Dandrieu écrit plus systématiquement les agréments sur les formules courantes, sa présence auprès de l'élève n'est plus nécessaire. Cette dernière édition est vraisemblablement destinée à un public plus large, à titre didactique, mais restreint au cercle privé, ce qui expliquerait le silence absolu des catalogues officiels et la suppression de la dédicace. Cette précision ornée est un cas d'école. Karl Philip Emanuel Bach en témoigne en 1753 :

On est en particulier saisi d'un préjugé malheureux à l'encontre des pièces de clavecin françaises, qui ont pourtant été une bonne école pour les exécutants, car c'est un pays qui s'est toujours distingué des autres de sa façon de jouer cohérente et juste. Tous les agréments indispensables y sont expressément indiqués ${ }^{62}$.

Ici, il faut rendre justice aux Français d'avoir mis tant de soin à écrire leurs morceaux. Les plus grands maitres de notre instrument on fait la même chose en Allemagne, mais avec moins d'excès. Et qui sait si ce n'est pas ce choix raisonné parmi le nombre des ornements qui a donné l'occasion aux Français de ne plus alourdir, aujourd'hui comme ils le faisaient auparavant, chaque note ou presque d'un ornement, qui finissait par cacher l'indispensable clarté et la noble simplicité du chant ${ }^{63}$ !

Afin de vendre leurs pièces de clavecin, les compositeurs tâchent de fournir un outil des plus pratiques pour les musiciens amateurs ou professionnels susceptibles d'acheter leurs œuvres. La gravure reste onéreuse, ce qui modifie le format et la présentation des recueils en fonction des finances des clavecinistes. Ainsi les tirages peuvent se multiplier, car au début, peu d'exemplaires sortent des presses. Les compositeurs profitent de ces retirages pour apporter des corrections qui parfois n'ont que des portées commerciales. Nous allons maintenant étudier ce pan de l'édition : la phase post-impression concernant les corrections, les lieux de vente, les rééditions légales ou piratées.

\section{La vente et la réédition}

31 Avec la technique de gravure, les plaques ayant servi au premier tirage peuvent être conservées et permettent de tirer les épreuves en fonction des besoins. La musicologue Anik Devriès explique en 1967 que les tirages étaient effectués par petits lots en fonction de la demande et des commandes. Les chiffres de 10,15 ou 20 exemplaires sont fréquemment cités dans les actes notariés comme base de commande aux marchands ou auteurs ${ }^{64}$. Une planche de cuivre ou d'étain autorisait un tirage total maximum de 250 exemplaires. Nous pouvons estimer grâce à l'inventaire après décès que la première édition de pièces de clavecin d'Anglebert se situait entre 300 et 400 exemplaires $^{65}$. Pour Rameau, selon la musicologue Sylvie Bouissou, peu d'exemplaires ont été imprimés, puisque peu d'exemplaires nous sont parvenus ${ }^{66}$. Cette pratique de gravure en lots successifs explique les constantes modifications, fussent-elles minimes, que l'on relève 
d'un exemplaire à l'autre, le compositeur et le graveur retouchant souvent leurs productions. Un simple rebouchage d'une gravure erronée ou démodée, mais aussi l'ajout de nouvelles corrections gravées suffiront là où nécessaire pour une réimpression. Encore de nos jours, il n'y a aucune entente générale entre bibliographes musicaux sur la question de savoir de qui constitue véritablement un tirage par opposition à une édition. Nous avons essayé d'unifier notre démarche, en contradiction parfois avec les termes utilisés par les musicologues, en dirigeant notre définition du tirage vers les exemplaires dont l'état distinct du livre premier, tel qu'il nous est parvenu, présente au moins une différence de gravure (musique ou titres) avec les autres exemplaires connus. La réédition est la réappropriation sur différentes plaques de gravures ou non, par un éditeur différent de l'original. Ci-dessous, le tableau récapitulatif du nombre de tirages et 
d'éditions de pièces de clavecin au tournant $d u \mathrm{XVII}^{\mathrm{e}}$ siècle nous indique que tous les livres de clavecin, à l'exception d'un, ont connu ces multiples sorties.

\begin{tabular}{|c|c|c|c|}
\hline Compositeurs & $\begin{array}{l}\text { Graveur } \\
\text { d'origine }\end{array}$ & $\begin{array}{l}\text { Nombre de tirages et } \\
\text { éditions }\end{array}$ & $\begin{array}{l}\text { Nombre d'exemplaires } \\
\text { retrouvés }\end{array}$ \\
\hline Chambonnières 1 & Jollain & 4 tirages (2 éditions ?) & 11 \\
\hline Chambonnières 2 & Jollain & 2 tirages (2 éditions ?) & 11 \\
\hline Lebègue 1 & Baillon & 6 tirages -2 éditions & 10 \\
\hline Lebègue 2 & Lesclop & $\begin{array}{l}2 \text { tirages de la } 1^{\text {re }}-2 \\
\text { éditions }\end{array}$ & 3 \\
\hline $\begin{array}{lll}\text { Jacquet de } & \text { La } \\
\text { Guerre } 1 & & \end{array}$ & Baussen & 2 tirages & 2 \\
\hline D'Anglebert & $\begin{array}{l}\text { Gillet et } \\
\text { Bonneuil }\end{array}$ & 4 éditions (5 probables) & 28 \\
\hline Marchand 1 & Roussel & 3 éditions & 4 \\
\hline Dieupart & Roger & 2 éditions & 8 \\
\hline Marchand 2 & Baussen & $\begin{array}{l}3 \text { éditions }-2 \text { tirages de } \\
\text { la } 2^{\mathrm{e}}\end{array}$ & 4 \\
\hline Clérambault & Roussel & 2 tirages & 2 \\
\hline Dandrieu & Roussel & 3 éditions & 4 \\
\hline Le Roux & Baussen & 2 éditions & 4 \\
\hline Rameau & Roussel & 2 éditions & 2 \\
\hline $\begin{array}{lll}\text { Jacquet de } & \text { La } \\
\text { Guerre 2 } & & \end{array}$ & Baussen & 1 édition & 1 \\
\hline Siret & Baussen & 2 tirages ( 1 avant 1713 ) & 2 \\
\hline
\end{tabular}

Ex. 7 : Tableau récapitulatif des tirages et éditions de pièces de clavecin

Comment expliquer l'exception du deuxième livre de Jacquet de La Guerre? Il semblerait qu'à 42 ans, 20 ans après son premier livre, son expérience lui a permis de présenter une édition ne nécessitant plus aucune retouche. Il reste étonnant qu'aucune réédition de ce livre n'ait été effectuée ou ne soit parvenue jusqu'à nous.

Le tableau ci-dessus indique qu'au $\mathrm{XVII}^{\mathrm{e}}$ siècle, les noms des graveurs sont beaucoup plus variés qu'au XVIII ${ }^{e}$ siècle. Chambonnières est le seul à éditer avec Jollain. Lebègue partage ses deux livres entre Baillon et Lesclop. Grâce à l'inventaire après décès d'Anglebert, nous savons que c'est Gillet et Bonneuil qui ont été chargés de la gravure des pièces de 
clavecin. Entre 1687 et 1710, deux graveurs, Baussen ${ }^{67}$ et Roussel ${ }^{68}$, se partagent le marché des pièces de clavecin, ce qui est peu face à la centaine de professionnels du métier comptés à Paris au XVIII ${ }^{\mathrm{e}}$ siècle ${ }^{69}$. Dieupart fait exception puisqu'il est le seul à publier à Londres avec Roger. Or, le choix du graveur modifie le paysage orné musical. Baussen propose toujours un travail fin et soigné. Chez Roussel, l'écriture semble moins précise et parfois plus grossière ${ }^{70}$. Ces petits détails interfèrent dans la lecture des partitions et donc son interprétation.
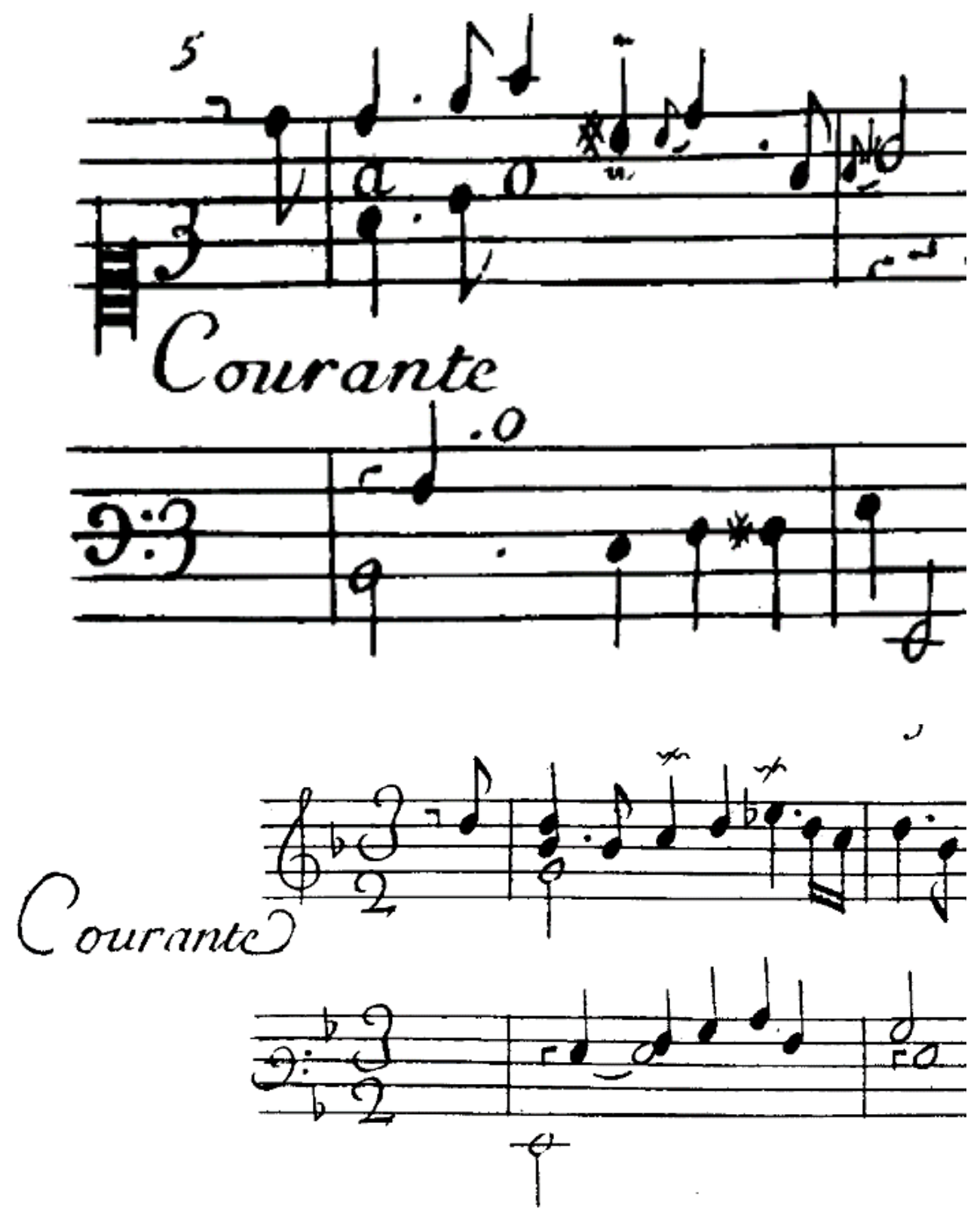

EX. 8 : COMPARAISON DE LA QUALITÉ DES gRAVURES : COURANTE 3 DU PREMIER LIVRE DE MARCHAND gRAVÉ PAR ROUSSEL ET COURANTE 12 DU SECOND LIVRE DE MARCHAND gRAVÉ PAR BAUSSEN

Les éditions de Roussel n'ont pas été corrigées et travaillées par les compositeurs après la première impression : Marchand, Clérambault et Rameau, sauf pour le premier livre de jeunesse de Dandrieu, 6 à 10 ans après. Chez Baussen, Jacquet de La Guerre (et Siret plus tard vers 1710) ont apporté des changements dans les ornements, notamment des ajouts d'agréments. Baussen semble être plus appliqué et plus à l'écoute des clavecinistes. Est-ce 
pour cela que Marchand l'a préféré à Roussel pour son second livre ? Le claveciniste a-t-il été déçu du travail de ce dernier pour le premier livre ? Ou encore, Roussel était-il moins cher que Baussen, s'occupant ainsi des musiciens jeunes et moins argentés? Jacquet de La Guerre, d'Anglebert, Marchand, Clérambault et Dandrieu. Jusqu'au début du XVII ${ }^{\mathrm{e}}$ siècle, elle avait plutôt lieu chez le graveur principal, mais cette pratique se délite au cours du XVIII ${ }^{\mathrm{e}}$ siècle, pour laisser place à la vente chez les libraires. Foucault l'imprimeur, à partir de 1702, devient un des revendeurs les plus courants des pièces de clavecin. En effet, il sera le seul marchand de musique français jusqu'en 1720 à assurer la diffusion de tout ce qui se publiera et se vendra en France dans le domaine de la musique, y compris les œuvres éditées par l'imprimeur Ballard ou des œuvres gravées à l'étranger. Il propose à la vente les pièces de Clérambault, Dandrieu, Le Roux et Rameau. Ballard va revendre, aux côtés de Foucault, plusieurs livres de clavecin dont le deuxième livre de Jacquet de La Guerre. Les deux ont été associés à partir de 1705 selon le musicologue Denis Herlin :

Un autre élément pour la datation de l'ouvrage [de Siret] est fourni par la double adresse de Ballard et Foucault qui figure sur la page de titre. Elle se trouve également dans les pièces de clavecin d'Élisabeth Jacquet de La Guerre de 1707. En effet, après plusieurs procès intentés par Ballard contre Foucault, il semble que ces deux éditeurs se soient associés seulement à partir de $1705^{71}$.

Un deuxième libraire moins prolifique, Ribou, propose dans sa boutique les deux recueils de Jacquet de La Guerre et celui de Dandrieu.

Ballard ne publie son premier livre de clavecin qu'en 1702, soit après 150 années d'activités dans l'édition musicale, et 33 ans après le premier livre gravé de l'histoire du clavecin. Cette nouveauté suppose en premier lieu un succès dont l'éditeur du roi voulait profiter. Celui-ci choisit une valeur sûre : la réédition des livres de Marchand, puis celle en 1703 du recueil d'Anglebert. Pour ce dernier ouvrage, Ballard ne prend aucun risque et récupère les planches d'origine héritées par la veuve d'Anglebert à la mort de son mari, ce qui reste la plus raisonnable des décisions puisque l'écriture ornée du claveciniste est impossible à reproduire avec la technique de caractères mobiles. L'inventaire après décès d'Anglebert de 1691 indique que les planches de gravure appartenaient toujours à la famille $^{72}$; Madeleine de Champagne, la veuve d'Anglebert, avait racheté à ses enfants les planches. L'édition Ballard apporte-t-elle un revenu supplémentaire à la famille? La veuve d'Anglebert avait déjà procédé, après la mort de son mari, à une deuxième édition des pièces de clavecin, soi-disant « Reveu et corrigé ». Or, l'analyse dévoile qu'il n'en est rien. C'est un subterfuge commercial. En 1702, lorsque Ballard réédite les deux livres de Marchand, quel contrat met-il en place avec le musicien? Les planches appartiennent normalement à l'auteur. Pourtant lors des rachats à l'occasion des successions des marchands, les planches gravées apparaissent dans les contrats. En 1736, le système changera puisque Leclerc innovera en ne commercialisant que des œuvres dont il a l'exclusivité. Le commerçant met alors en place un système novateur où les planches appartiennent de moitié au marchand et au compositeur ${ }^{73}$.

En 1707, Ballard publie, un petit livre de vingt-huit pages, sous le titre : Pièces choisies / pour le clavecin / de différents auteurs. Aucune adresse, ni aucune date n'est ajoutée à ce titre, qui a ainsi toute l'apparence d'un faux titre. Le volume contient seulement 10 pièces et les noms des auteurs ne sont pas donnés. Il est probable que nous avons là une de ces publications subreptices, établies sans la participation des auteurs, sur les manuscrits des ouvrages dont la réputation courait le monde. Des pièces de Couperin et une pièce inédite 
de Marchand font partie de ce recueil ${ }^{74}$. La visée commerciale est clairement définie. Le livre est imprimé avec les caractères ordinaires des Ballard, assez grossièrement et assez mal lisible. En conséquence, les agréments sont moins variés, moins nets, simplifiés et très souvent supprimés. On mesure ici, les raisons du triomphe, presque achevé à ce moment déjà, de la gravure : raisons qui ont fait la décadence de l'antique maison des imprimeurs du roi pour la musique.

Dans le même esprit, dès le tournant du siècle, le piratage des éditions de pièces de clavecin se met en place à Amsterdam avec l'imprimeur Roger. Cette pratique nous permet de constater quels étaient les compositeurs en vogue en Europe à cette époque et susceptibles d'intéresser un large public et de permettre à l'éditeur de faire des profits. Néanmoins, nous ne devons pas faire de généralités, car l'absence de piratage de certains compositeurs peut s'expliquer autrement que par un manque d'intérêt pour ces compositeurs. Le musicologue Bruce Gustafson s'étonne, par exemple, de l'absence de copie amsterdamoise des pièces de Chambonnières et explique celle-ci par le coût de l'édition et la présence des manuscrits :

Les éditions de Lebègue et d'Anglebert étaient piratées à la fin du siècle à Amsterdam, contrairement aux pièces de Chambonnières. Cela s'explique sûrement non pas par un manque de popularité des œuvres, mais par le fait que la musique de Chambonnières était plus disponible. La musique imprimée coûtait cher, et les œuvres de Chambonnières circulaient sous forme de manuscrits moins chers, à la différence de celles de Lebègue et d'Anglebert ${ }^{75}$.

40 Le premier claveciniste français à être copié par Roger est Lebègue en 1697, viennent ensuite Marchand vers 1701, d'Anglebert en 1705 et Le Roux en dernier, vers 1707. La vogue française ne durera que 10 ans. Chez Roger à Amsterdam, le livre de Marchand est vendu 1,4 florins $^{76}$. D'Anglebert y est vendu à 7 florins, le premier livre de Lebègue à 6 florins et le second à 5 florins. Enfin, le recueil de Dieupart qui vit à Londres voit le jour à Amsterdam uniquement grâce à l'imprimeur Roger. Il coûte 9 florins dans les catalogues de Roger en $1716^{77}$.

\section{Conclusion}

$41 \mathrm{Au} \mathrm{XVIII}^{\mathrm{e}}$ siècle les copies des livres de pièces de clavecin à Amsterdam par Roger démontrent le succès croissant de ces œuvres dont la présentation typiquement française répond à un souci commercial fort. Même l'imprimeur du roi qui avait tourné le dos pendant près de 150 ans à l'édition de pièces de clavecin, se voit obligé de récupérer une part de ce marché fructueux. Il est alors contraint de racheter les planches gravées puisque sa technique d'impression est inadaptée à l'écriture musicale pour clavecin dès le tournant du XVII siècle. Le commerce se fait autour des mêmes individus que ce soit pour la gravure ou pour la vente.

42 La première édition de 1670 de Chambonnières a donc mis en place un système possible grâce au développement de la gravure. Frontispice, dédicace, table des agréments, écriture ornée, arrangements nouveaux, tout ceci constitue le contenu des recueils allant de 1670 à 1707 et répond à des nécessités commerciales : remercier les mécènes sans qui l'impression n'aurait pas été aussi soignée et attirer des amateurs riches afin de pouvoir vendre le produit. Ce produit est sans cesse amélioré grâce à l'expérience acquise depuis 1670 et grâce aux répercussions des premiers tirages. L'économie tient une place primordiale dans l'édition des pièces de clavecin. Les musiciens aujourd'hui doivent 
prendre conscience de ce fait, afin de préciser la place du support éditorial dans la pratique, notamment par rapport au style musical de l'époque qu'il nous communique.

\section{BIBLIOGRAPHIE}

\section{Partitions}

ANGLEBERT Jean-Henry D', Pièces de clavecin, Paris, l'Auteur, 1689.

CHAMPION DE CHAMBONNIÈRES Jacques, Les pièces de clavessin, Livre premier, Paris, Jollain, 1670.

CHARPENTIER Marc Antoine, Motets melez de symphonie, Paris, J. Édouard, 1709.

DANDRIEU Jean-François, Livre de clavecin, Paris, Ribou, Foucault, l'Auteur, [1704].

DIEUPART François, Six suittes de clavecin, Amsterdam, Roger, [1701].

JACQUET DE LA GUERRE Élisabeth-Claude, Pièces de clavecin premier livre, Paris, l'Auteur, De Baussen, [1687].

JACQUET DE LA GUERRE Élisabeth-Claude, Pièces de Clavecin qui peuvent se joüer sur le viollon, Paris, l'Auteur, Foucault, Ribou, Ballard, 1707.

JACQUET DE LA GUERRE Élisabeth-Claude, Pièces de clavecin, Deuxième livre, Paris, Baillon, l'Auteur, 1687.

LEBÈGUE Nicolas-Antoine, Pièces de clavecin, Premier livre, Paris, Baillon, l'Auteur, 1677.

LE ROUX Gaspard, Pièces de clavessin, Paris, Foucault, 1705.

MARCHAND Louis, Premier Livre de pièces de clavecin, Paris, Ballard, [1699].

MARCHAND Louis, Second Livre de pièces de clavecin, Paris, Ballard, 1702.

PAULIN Frédéric-Hubert, Airs sérieux et à boire, Paris, Pierre Ribout, 1705.

RAMEAU Jean-Philippe, Premier Livre de pièces de clavecin, Paris, l'Auteur, Roussel, 1706.

RAMEAU Jean-Philippe, Pièces de clavecin avec une méthode pour la mechanique des doigts, Paris, Boivin, Hochereau, l'Auteur, 1724.

ROBERDAY François, Fugues et Caprices à quatre parties mises en partition, Paris, Vve Sanlecque, J. Hanocq \& J. Laisné, 1660.

\section{Sources avant 1800}

Archives nationales de France, Minutier central, Étude LIII, 105, partage de la succession de JeanHenry d'Anglebert, 23 novembre 1691.

Le Mercure Galant, novembre 1686.

ВACH Carl Philipp Emanuel, Versuch über die wahre Art das Clavier zu spielen, Berlin, Henning, 1753; rééd. Paris, J.C. Lattès, 1979. 
GARSAULT François-Alexandre-Pierre de, Notionnaire ou mamorial raisonné de ce qu'il y a d'utile et d'interessant, Paris, Guillaume Desprez, 1761.

RIPA Cesare, Iconologie..., Paris, Baudoin, 1643.

SEGRAIS Jean Regnault DE, Segraisiana ou melange d'histoire et de litterature. Recueilli des entretiens de monsieur de Segrais de l'Academie françoise, Paris, Compagnie des libraires associés, 1721.

\section{Bibliographie après 1800}

BENOÎT Marcelle, Les musiciens du roi de France (1661-1733), Paris, PUF, 1982.

BERNHARDT Vincent, Une réflexion sur l'interprétation de la musique pour clavier de J.S. Bach : la problématique de l'ornementation, en ligne.

BouIssou Sylvie, "De la notation abrégée et réduite, et de ses enjeux économiques », Histoire de la notation de l'époque baroque à nos jours, Clamecy, Minerve, 2005.

BoUISsou Sylvie, Jean-Philippe Rameau, Paris, Fayard, 2014.

BROSSE Jean-Patrice, Le clavecin du Roi Soleil, Paris, Bleu nuit, 2011.

COUTURE Johanne, « Nouvelles perspectives sur le répertoire français pour clavecin au XVII siècle ", Recherches sur la musique française classique, vol. 31, 2007, p. 3-16.

CYPESS Rebecca, "Chambonnières, Jollain and the first engraving of harpsichord music », Early Music, vol. 35, $\mathrm{n}^{\circ}$ 4, 2007, p. 539-553.

DART Thurston, « Préface », BRUNOLD Paul (ed.), Pièces de clavecin de Louis-Nicolas Clérambault, Monaco, L’Oiseau-lyre, [années 1930] ; rééd. Thurston Dart, 1964 ; rééd. 2004.

DEVRIÈs Anik, Éditions et commerce de la musique gravée à Paris dans la première moitié du XVIII siècle, Le Boivin, Les Leclerc, Genève, 1976.

FRANÇOIS-SAPPEY Brigitte, « L'œuvre de clavecin de Jean-François Dandrieu (1682-1738), Trois livres de jeunesse », Recherches, vol. 14, 1974, p. 154-235.

GUILLO Laurent, « Les Ballard : Imprimeurs du Roi pour la musique ou imprimeurs de la musique du Roi ? », DURON Jean (dir.), Le Prince et la musique, Les passions musicales de Louis XIV, Wavre, Mardaga, p. 275-288.

GUSTAFSON Bruce, French Harpsichord music of the 17th Century, Ann Arbor, UMI Research Press, 1979.

HERLIN Denis, « Introduction », Pièces de clavecin dédiées à Monsieur Couperin de Siret, Paris, Paris, Société française de musicologie, 2001

HILL John Walter, Baroque Music, Music in Western Europe 1580-1750, University of Illinois, UrbanaChampaign, W.W. Norton \& Company, 2004.

HUYGHENS Christian, CEuvres complètes, La Haye, 1888.

HUYGHENS Constantin, Correspondance et œuvres musicales, Leyde, éd. Jongkbloet-Land, 1882.

KOCEVAR Érik, «Jean-Henry d'Anglebert ou Jean Henry, dit Anglebert? Mise au point sur le véritable nom des d'Anglebert à la lumière de documents d'archives inédits ", Ostinato, $\mathrm{n}^{\circ} 8 / 9$, 1997, p. 67-86.

LESURE François, « Une querelle sur le jeu de la viole en 1688 : Jean Rousseau contre Demachy », Revue de musicologie, t. 46, 1960. 
LESURE François, Bibliographie des éditions musicales publiées par Estienne Roger et Michel-Charles Le Cène (Amsterdam 1696-1743), Paris, Heugel, 1969.

MORONEY Davitt, « Chambonnières and his "Belle manière” ", SCHNEIDER Herbert \& LA GORCE Jérôme DE (dir.), Jean-Baptiste Lully, actes du colloque, Laaber, Laaber-Verlag, 1990.

QUITTARD Henri, « Un claveciniste français du XVII ${ }^{\mathrm{e}}$ siècle, Jacques Champion de Chambonnières ", Tribune de Saint-Gervais, vol. 2, 1901.

STAUFFER George B., The World of Baroque Music, Bloomington, Indiana University Press, 2006.

TALLEMANT DeS RÉAuX Gédéon, « Contes de bestes », Historiettes, Paris, 1834-1835, rééd. Paris,

A. Adam, 1960-1961.

TESSIER André, « Attribution à Couperin le Grand d'une pièce anonyme d'un recueil de Ballard », Revue de musicologie, t. 2, 1922, p. 69-78.

Livrets de disques compacts

BAUMONT Olivier, « La source de la belle manière du toucher », Les Pièces de Clavecin Jacques Champion de Chambonnières, Olivier Baumont, disque compact, AS Musique, 2003.

FLINT Karen, « D'exquises mélodies et le plus beau toucher », Les pièces de Clavessin, Jacques Champion de Chambonnières, Karen Flint, disque compact, Plectra, 2010.

MORRIER Denis, « Louis Marchand, un honnête homme?» Euvres pour clavier, Marchand, Laurent Stewart, disque compact, Hortus, 2006.

SEMPÉ Skip, « Skip Sempé en conversation avec Thomas Mace », Paris, Printemps 1992, Jacques Champion de Chambonnières, Pièces de clavecin, Skip Sempé, Harmonia Mundi, 1992.

\section{NOTES}

1. Ce manuscrit est consacré à de la musique anglaise, italienne et néerlandaise avec les noms de Byrd, Cornet, Farnaby, Gibbons, Tallis, Ferrabosco et Sweelinck, et au milieu desquels se glisse celui de Champion, père de Chambonnières, comme auteur d'une Fantasia, d'une Pavane, et d'une Galliard. Ce livre porte le nom de son propriétaire, le Docteur John Bull. Les dates qui y sont mentionnées sont celles de 1621 et 1628.

2. QUITTARD Henri, «Un claveciniste français du $\mathrm{XVII}^{\mathrm{e}}$ siècle, Jacques Champion de Chambonnières ", Tribune de Saint-Gervais, vol. 2, 1901, p. 37.

3. Cf. BENôiT Marcelle, Les musiciens du roi de France (1661-1733), Paris, PUF, 1982, p. 72.

4. Cf. COUTURE Johanne, « Nouvelles perspectives sur le répertoire français pour clavecin au XVII ${ }^{e}$ siècle ", Recherches sur la musique française classique, vol. 31, 2007, p. 3-16.

5. Cf. Bouissou Sylvie, "De la notation abrégée et réduite, et de ses enjeux économiques. », Histoire de la notation de l'époque baroque à nos jours, Clamecy, Minerve, 2005.

6. ROBERDAY François, Fugues et Caprices à quatre parties mises en partition, Paris, Vve Sanlecque, J. Hanocq \& J. Laisné, 1660.

7. Cf. GUiLLo Laurent, « Les Ballard: Imprimeurs du Roi pour la musique ou imprimeurs de la musique du Roi ?», DURON Jean (dir.), Le Prince et la musique, Les passions musicales de Louis XIV, Wavre, Mardaga, p. 275-288. 
8. « Recherches de Walter J. Ong sur la relation entre les traditions orales et écrites [...] : L'impression de la langue écrite change l'évolution de l'alphabétisation et peut se calquer sur le transfert, initié par Chambonnières, d'une tradition strictement orale et manuscrite à la transmission et la propagation par la partition imprimée.» "Walter J. Ong's investigation [ONG, W.J., Orality and literacy : the technologizing of the word, London, 1982] of the relationship between oral and written traditions [....] the printing of the written word changes the nature of literacy may be mapped easily onto the shift, initiated by Chambonnières, from a strictly oral and manuscript tradition to transmission and dissemination trough print." (CYPRESS, Rebecca, "Chambonnières, Jollain and the first engraving of harpsichord music », Early Music, vol. 35, $\mathrm{n}^{\circ}$ 4, 2007, p. 539-553, ici p. 544.)

9. Le manuscrit Oldham ou London Couperin manuscript daté entre 1650 et 1661 ne contient pas moins de 14 pièces du livre de Chambonnières publié en 1670.

10. CHAMPION DE CHAMBONNIÈRES, Jacques, Les pièces de clavessin, Livre premier, Paris, Jollain, 1670.

11. BAUMONT Olivier, "La source de la belle manière du toucher ", Les Pièces de Clavecin Jacques Champion de Chambonnières, disque compact, AS Musique, 2003, p. 17.

12. FLINT Karen, "D'exquises mélodies et le plus beau toucher ", Les pièces de Clavessin, Jacques Champion de Chambonnières, disque compact, Plectra, 2010, p. 19.

13. KOCEVAR Érik, "Jean-Henry d'Anglebert ou Jean Henry, dit Anglebert? Mise au point sur le véritable nom des d'Anglebert à la lumière de documents d'archives inédits ", Ostinato, n' 8/9, 1997, p. 67-86.

14. En effet, vers la fin du XVII ${ }^{e}$ siècle, la politique de Louis XIV semble changer pour des raisons financières. En 1696, les guerres victorieuses du roi ont coûté cher. Aussi a-t-on recours à un expédient pour renflouer les caisses du royaume : une suite d'édits permet l'anoblissement de personnes qui ont été distinguées pour leur mérite et contre vingt livres. Couperin est ainsi anobli, et avec lui ses confrères de la Musique du roi, séduits par cette possibilité de promotion: Lalande, Buterne, d'Anglebert fils, Beaumont, André et Jacques Danican Philidor. Son blason va ainsi figurer dans l'Armoirial des Généralités que Charles d'Hozier le généalogiste du roi, a commencé à établir à partir de l'année 1696.

15. BROSSE Jean-Patrice, Le clavecin du Roi Soleil, Paris, Bleu nuit, 2011, p. 93.

16. Cf. LESURE François, « Une querelle sur le jeu de la viole en 1688 : Jean Rousseau contre Demachy. », Revue de musicologie, t. 46, 1960, p. 184.

17. SEMPÉ Skip, «Skip Sempé en conversation avec Thomas Mace », Paris, Printemps 1992, Jacques Champion de Chambonnières, Pièces de clavecin, Skip Sempé, Harmonia Mundi, 1992, p. 12.

18. Cf. HUYGHENS Christian, «Lettre du 20 décembre 1660 », CEuvres complètes, La Haye, 1888, p. 138. Christiaan déclara brièvement le 20 décembre $1660:$ : Vu Chambonnières qui joua du clavecin et chanta un air de sa façon qui ne me sembla que médiocre ». Le 14 septembre 1662, il écrivit à nouveau, d'une façon bien peu charitable d'ailleurs: "L'estat du marquis de Chambonnières me feroit pitié s'il n'avoit pas si fort fait l'entendu auparavant. La dernière fois que je le vis, il vouloit encore me faire accroire qu'il ne jouoit plus du clavecin et le voilà misérable maintenant s'il ne scavoit pas ce mestier ».

19. SEGRAIS Jean Regnault DE, Segraisiana ou melange d'histoire et de litterature. Recueilli des entretiens de monsieur de Segrais de l'Academie françoise, Paris, Compagnie des libraires associés, 1721, p. 60. 
20. TAllemant DES RÉAuX Gédéon, «Contes de bestes », Historiettes, Paris, 1834-1835, rééd. Paris, A. Adam, 1960-1961, p. 16.

21. «Je vous advertiray que le très illustre Sieur de Chambonnières [...] se trouve ici desgoutté de se veoir ostée par le bas et mauvais mesnage qui règne en cette cour une pension d'environ mi escus par an, qu'il y auroit moyen d'en chevir, s'il trouvoit un prince digne amateur de sa science et capable de le faire vivre avec un peu d'honneur, comme il a toujours faict icy... ». (HUYGHENS Constantin, « Lettre XLIV », Correspondance et œuvres musicales, Leyde, éd. Jongkbloet-Land, 1882, p. 34.)

22. ANGLEBERT Jean-Henry D', Pièces de clavecin, Paris, l'Auteur, 1689.

23. Le Mercure Galant, novembre 1686.

24. MARChAND Louis, Premier livre de pièces de clavecin, Paris, Ballard, [1699].

25. Les changements d'éditeurs de Marchand sont sûrement en lien avec son caractère difficile ou à ses problèmes financiers qui commencent.

26. MORRIER Denis, "Louis Marchand, un honnête homme?", Euvres pour clavier, Marchand, Laurent Stewart, disque compact, Hortus, 2006, p. 3.

27. DANDRIEU Jean-François, Livre de clavecin, Paris, Ribou, Foucault, l'Auteur, [1704].

28. Cf. FRANÇOIS-SAPPEY, Brigitte, «L'œuvre de clavecin de Jean-François Dandrieu (1682-1738), Trois livres de jeunesse », Recherches, $n^{\circ} 14,1974$, p. 154-235, p. 163.

29. RAMEAU, Jean-Philippe, Premier livre de pièces de clavecin, Paris, l'Auteur, Roussel, 1706.

30. LE ROUX, Gaspard, Pièces de clavessin, Paris, Foucault, 1705.

31. Le médaillon chez Dandrieu représente un bœuf, écusson de Monsieur Robert chevalier de Septeuil à qui le claveciniste dédicace son œuvre.

32. Cf. Airs sérieux et à boire de Paulin en 1705 et les Motets melez de Symphonie de Charpentier en 1609.

33. RIPA Cesare, Iconologie..., Paris, Baudoin, 1643, p. 123-124.

34. Ibid.

35. DIEUPART François, Six suittes de clavecin, Amsterdam, Roger, [1701].

36. LE ROUX, Pièces de clavessin.

37. JACQUET DE LA GUERRE Élisabeth-Claude, Pièces de Clavecin qui peuvent se joüer sur le viollon , Paris, l'Auteur, Foucault, Ribou, Ballard, 1707.

38. GARSAULT François-Alexandre-Pierre DE, «Clavecin », Notionnaire ou mamorial raisonné de ce qu'il y a d'utile et d'interessant, Paris, Guillaume Desprez, 1761, p. 640.

39. Il en est de même pour les organistes, rappelons que la première gravure que nous connaissons est celle d'un organiste : Fugues et Caprices à quatre parties mises en partition par François Roberday, en 1660, chez la Veuve Sanlecque, J. Hanocq \& J. Laisné.

40. Certains prix sont inconnus aujourd'hui à la date de publication. Le coût indiqué est plus tardif (de 1734 à 1742), durant une période où les catalogues sont plus précis et sont parvenus jusqu'à nous. Cf. Troisième partie : La vente et la réédition.

41. La livre en tant que pièce de monnaie n'existe pas. Le terme « livre » correspond à une unité de poids dans laquelle étaient taillés un certain nombre de flans pour en faire des pièces (au temps de Charlemagne, la livre de métal était divisée en 20 parties égales pour en faire 20 sols). Une livre égale 489,5 grammes. 
42. DART Thurston, "Préface», BRUNOLD Paul (ed.), Pièces de clavecin de Louis-Nicolas Clérambault, rééd. Thurston DART, Monaco, L'Oiseau-lyre, 1964 ; rééd. 2004, p. 1.

43. «Vraisemblablement, il est possible que la raison pour laquelle Jollain ait imprimé le travail de Chambonnières simultanément en deux volumes séparés était de les rendre plus abordables, et ainsi plus accessibles à l'achat. " "Furthermore, it is possible that the reason Jollain printed Chambonnières's works simultaneously in two separate volumes was to make them more affordable, and therefore more accessible for purchase. Given these circumstances, it is unlikely that Chambonnières expected to gain financially from his publications." (CYPRESS, Rebecca, «Chambonnières, Jollain and the first engraving of harpsichord music », p. 545.) «Thurston Dart suppose que Jollain sépara le seul volume en deux, de peur que l'unique large tome soit trop cher à la vente. " "Thurston Dart speculated that Jollain broke a single volume in two, lest the one large tome prove too expensive to sell." (GUSTAFSON Bruce, French Harpsichord music of the 17th Century, Ann Arbor, UMI Research Press, 1979, p. 131.)

44. DEVRIÈs Anik, Éditions et commerce de la musique gravée à Paris dans la première moitié du XVIII ${ }^{e}$ siècle, Le Boivin, Les Leclerc, Genève, 1976, p. 53.

45. Ibid., p. 133.

46. Ibid., p. 160.

47. Ibid., p. 208.

48. Ibid., p. 165.

49. Ibid., p. 243.

50. "Beginning in the late 1680s, as significant number of the composers of important French harpsichord music did not have appointments at the royal court. [...] Even when a composer held a court position, his harpsichord music was now commercially printed for amateurs of means and education, rather than preserved only manuscript." (HILL John Walter, Baroque Music, Music in Western Europe 1580-1750, University of Illinois, Urbana-Champaign, W.W. Norton \& Company, 2004, p. 440.)

51. Premier tirage de 1702 : F-Pn Rés. Vmc 174.

52. BERNHARDT Vincent, Une réflexion sur l'interprétation de la musique pour clavier de J.S. Bach : la problématique de l'ornementation, en ligne, p. 4-5.

53. "The revisions which he carried out on his own works can be studied by comparing the manuscript versions with the texts he finally published in 1670; the act of revision was for him almost always a process of simplification, a paring down of all complications to produce a lean and purified style [....] (Some scholars have even felt it strange that he should have published his "less interesting" pieces, but this is to confuse the complex with the interesting)." (MORONEY Davitt, "Chambonnières and his "Belle manière" ", SCHNEIDER Herbert \& LA GORCE Jérôme DE (dir.), Jean-Baptiste Lully, actes du colloque, Laaber, Laaber-Verlag, 1990, p. 207.

54. LebèGUE Nicolas-Antoine, Pièces de clavecin, Premier livre, Paris, Baillon, l'Auteur, 1677.

55. Deuxième édition par Roger à Amsterdam vers 1697, exemplaire de la bibliothèque de l'université de Lund en Suède : Lunds Akademiska Kapell, J.H. Englehart's donation n 24 .

56. Dans un des exemplaires du premier livre de Lebègue, une réponse de ce dernier, à une lettre d'un amateur anglais William Dundass a été envoyée en 1684 après une demande d'explication sur l'interprétation préludes non mesurées. Lettre du 3 juillet 1684 pour M. William Dundass, Londres, British Museum Library, K 10a 14. 
57. Ces pourcentages sont issus du calcul du nombre d'agréments dans les pièces de clavecin par rapport au nombre de notes.

58. LEBÈGUE Nicolas-Antoine, Pièces de clavecin, Deuxième livre, Paris, Baillon, l'Auteur, 1687.

59. JACQUET DE LA GUERRE Élisabeth-Claude, Pièces de clavecin premier livre, Paris, l'Auteur, De Baussen, [1687].

60. MARCHAND Louis, Second livre de pièces de clavecin, Paris, Ballard, 1702.

61. Nous comparons avec le deuxième livre de Rameau, bien plus tardif que le premier car daté de 1724 : RAMEAU Jean-Philippe, Pièces de clavecin avec une méthode pour la mechanique des doigts, Paris, Boivin, Hochereau, l'Auteur, 1724.

62. BACH Carl Philipp Emanuel, Versuch über die wahre Art das Clavier zu spielen, Berlin, Henning, 1753; rééd. Paris, J.C. Lattès, 1979, p. 28.

63. Ibid., p. 94.

64. DEVRIÈs, Éditions et commerce de la musique gravée à Paris, p. 7.

65. Archives nationales de France, Minutier central, Étude LIII, 105, partage de la succession de Jean-Henry d'Anglebert, 23 novembre 1691. Le partage de succession précise qu'il existait une série d'exemplaires en blanc, c'est-à-dire non reliés, qui s'élevait à deux-cent vingt-cinq exemplaires. Les exemplaires furent répartis entre Madeleine de Champagne la veuve de d'Anglebert de la manière suivante: 113 pour elle-même et 14 exemplaires pour chacun des huit enfants. Cette précision permet d'évaluer à 266 le nombre d'exemplaires restants après un an et demi de publication. Le tirage initial devait donc se situer entre 300 et 400 exemplaires.

66. «Rameau ne souhaitait faire des frais inutiles pour la publication de son premier livre. D'ailleurs, il n'en sera tiré qu'un petit nombre puisqu'aujourd'hui on ne recense de cette première édition que deux exemplaires, l'un à Paris, l'autre à Bordeaux. » (Bouissou Sylvie, Jean-Philippe Rameau, Paris, Fayard, 2014, p. 62.)

67. Le graveur Baussen a déjà travaillé pour les organistes Gigault et Grigny. Ancien musicien de Mademoiselle de Guise, Baussen exerça son activité à partir de 1685 avec la gravure du troisième livre d'orgue de Lebègue. Initié à cet art par son beau-père, Pierre Baillon, dont il avait épousé la fille en 1681, il fut l'un des plus importants graveurs de musique de 1685 à 1716, date probable de son décès. En 1711, il prit en apprentissage Louis Hue, qui allait devenir à son tour, le graveur le plus productif de sa génération. Baussen a gravé 5 ouvrages pour le clavecin.

68. Roussel est graveur, éditeur et marchand d'estampes et de cartes géographiques, également graveur de musique. Établi dès 1682 à Paris, il s'y marie. Il est en procès pour contrefaçon de planche en juin 1699 mais encore en activité en 1725.

69. L'état français permettait un certain commerce (même s'il était forcément limité) des textes et œuvres publiés à Paris et destinées au public de la capitale, qui pouvaient ainsi toucher aussi la bourgeoisie et la noblesse de campagne grâce au réseau routier fortement amélioré par Colbert à la demande de Louis XIV et permettant une bien meilleure circulation des voyageurs - et donc des livres. On compte, au XVIII siècle, jusqu'à 150 graveurs travaillant à Paris qui devient le centre musical européen de l'imprimerie comme en conclut le musicologue George Stauffer en 2006: "The high quality and beauty of the engravings, the excellent publicity offered by the engravers and music organizations, and the wide distribution of the finished collections made in Paris the European center for music printing" 
(STAUFFER George B., The world of baroque music, Bloomington, Indiana University Press, 2006, p. 119.)

70. Cf. essentiellement la gravure du livre de Clérambault ou le premier livre de Marchand.

71. HERLIN, Denis, «Introduction», Pièces de clavecin dédiées à Monsieur Couperin de Siret, Paris, Société Française de Musicologie, 2001, p. XI. Siret a publié son premier livre de pièces de clavecin vers 1710, la date reste inconnue. En 1976, la musicologue Anik Devrès écrit que « Foucault s'associera aussi entre 1709 et 1715 avec la belle-sœur de Christophe Ballard, la Veuve Pierre Ballard. » (DEVRIÈs, Éditions et commerce de la musique gravée à Paris, p. 16.)

72. Archives nationales de France, Minutier central, Étude LIII, 104, inventaire après décès de d'Anglebert, 8 mai 1691.

73. Cf. DEVRIÈs, Éditions et commerce de la musique gravée à Paris, p. 57.

74. La Vénitienne de Marchand, qui est inédite et L'abeille, les Nonettes, la Diane, la Florentine et la Badine de François Couperin qui seront publiées par l'auteur en 1713 dans son premier livre. En 1922, le musicologue André Tessier attribue la deuxième Badine (pièce inédite) de ce recueil à Marchand, mais aucun autre musicologue n'avance cette théorie, nous n'avons donc pas ajouté cette œuvre à la liste. TESSIER, André, "Attribution à Couperin le Grand d'une pièce anonyme d'un recueil de Ballard ", Revue de musicologie 2 (1922), p. 69-78.

75. "Lebègue's and d'Anglebert's prints were pirated in Amsterdam at the turn of the century, but Chambonnières' pieces were not. The explanation may lie less with any lack of popularity of the works than with the ready availability of Chambonnières'music. Printed music was expensive and Chambonnières' pieces were widely circulated in cheaper manuscripts, unlike those of Lebègue and d'Anglebert." (GUSTAFSON, French Harpsichord music of the $17^{\text {th }}$ Century, p. 133.)

76. LESURE François, Bibliographie des éditions musicales publiées par Estienne Roger et MichelCharles Le Cène (Amsterdam 1696-1743), Paris, Heugel, 1969, p. 162.

77. Ibid., p. 65 et 162.

\section{RÉSUMÉS}

Alors que les manuscrits de pièces de clavecin circulent en Europe dès les années 1620, le premier livre édité en France voit le jour cinquante ans plus tard, en 1670. À partir de cette date, les recueils pour cet instrument en soliste vont se multiplier. De 1670 à 1707, neuf compositeurs vont publier à Paris à compte d'auteur : Chambonnières (1670), Lebègue (1677 et 1687), Jacquet de la Guerre (1687 et 1707), d'Anglebert (1689), Marchand (1699 et 1702), Clérambault (1704), Dandrieu (1704), Le Roux (1705) et Rameau (1706). Nous tenterons d'expliquer l'apparition de cette nouvelle source musicale et les raisons de son développement au début du XviII ${ }^{\mathrm{e}}$ siècle. Le progrès technique mais surtout les intérêts économiques tiennent une place primordiale dans l'histoire de l'édition du clavecin. Chaque recueil est conçu dans des circonstances particulières où le 
compositeur, en fonction de sa situation pécuniaire et de ses choix financiers, va mettre au jour un format d'œuvres, devenu aujourd'hui une source musicale principale.

\section{INDEX}

Mots-clés : clavecin, édition, ornement, agrément, économie, prix, gravure, tirage, réédition, Ballard, Roger, Foucault, Chambonnières, Lebègue, Jacquet de la Guerre, d'Anglebert, Marchand, Clérambault, Dandrieu, Le Roux, Rameau, dédicace, mécène

\section{AUTEUR}

\section{CHLOÉ DOS REIS}

Professeure agrégée d'éducation musicale, docteure et claveciniste, Chloé Dos Reis enseigne à Sorbonne Université. Ses recherches portent sur la musique française pour clavecin des XVII et XVIII ${ }^{\mathrm{e}}$ siècles (approche herméneutique, esthétique, esthésique, poiétique, historique...), sur ses transferts et ses influences et leurs réceptions aux siècles suivants (historiographie, factures, enseignements, performance studies \& practice, filmographie). 